\title{
Cavity-Based Single-Photon Sources
}

\author{
Axel Kuhn* and Daniel Ljunggren ${ }^{\dagger}$ \\ University of Oxford, Clarendon Laboratory, Parks Road, OX1 3PU, United Kingdom
}

(Dated: January 05, 2010)

\begin{abstract}
We introduce the basic concepts and characteristic properties of single-photon emitters based on resonator effects in optical cavities, and provide a review of the most prominent implementations. First we discuss the elementary principles of cavity quantum electrodynamics, which determine how single quantum systems couple to the quantised field modes of optical resonators, and then show how to exploit these principles in order to generate single photons on demand. Furthermore, we elucidate how to study the properties of the photons, namely their singleness and their spectral characteristics. In particular, it is explained how to use a Hanbury-Brown \& Twiss interferometer to determine whether the photons arrive one-by-one, and how to probe their mutual indistinguishability with twophoton interference experiments of the Hong-Ou-Mandel type. Finally, we present a classification scheme applicable to most cavity-based photon emitters realised to date. This scheme allows us to assess the pros and cons of the most significant approaches - in particular with regard to photonic quantum computing and quantum communication, which are among the most promising applications to date.
\end{abstract}

\section{Contents}

\section{Introduction}

II. Atom-photon interaction in resonators

A. Field quantisation in cavity QED

B. Two-level atom

C. Three-level atom

D. Cavity-coupling regimes

III. Single-photon emission

A. Cavity-enhanced spontaneous emission

B. Steady-state evolution in the bad-cavity regime

C. Dynamic evolution in the strong-coupling regime

D. Arbitrary photon shaping

\section{Single-photon characterisation}

A. Statistics: photons one-by-one

B. Indistinguishability: the photon identity

Two-photon interference

Step-by-step analysis

Identical and distinguishable photons

Frequency jitter

Time jitter

\section{Routes to single-photon sources}

A. Neutral single atoms

B. Trapped single ions

C. Artificial atoms in solid-state

\section{Future directions}

\footnotetext{
*Corresponding author: axel.kuhn@physics.ox.ac.uk

†Current affiliation: Dept. of Applied Physics, KTH - Royal Institute of Technology, 10691 Stockholm, Sweden. Email: daniellj@ kth.se
}

Acknowledgments

References

\section{INTRODUCTION}

The ultimate control of individual quantum systems is a key element in emerging topics of modern physics, such as quantum information processing. Here, the achievable degree of control relies on the ability to arbitrarily address, manipulate, and couple individual physical entities, like single atoms or single photons, which are used as information carriers. For instance, the interfacing of atoms and photons, the storage and retrieval of single photons, and the mapping of quantum states between distant entities, all constitute essential building blocks of future quantum communication networks and quantum information processors [1]. Ideally, such systems are composed of individual nodes acting as quantum gates or memories, with optical links in between that allow for the entanglement or teleportation of their quantum states, or for optical quantum information processing using light travelling between the nodes [2]. With individual photons acting as messengers in these networks, wide efforts are being undertaken that focus on the production and characterisation of single photons. Applications relying on the availability of single photons include quantum cryptography, optical quantum computing, light-matter entanglement, and atom-photon state mapping, which all have successfully been demonstrated in the past few years.

In this article, we restrict ourselves to review the concepts, characteristic properties, and major implementations of state-of-the-art single-photon sources based on single emitters in cavities. These have all the potential to meet the requirements of optical quantum computing and quantum networking schemes, namely

- deterministic single-photon emission with unit effi- 
ciency,

- directed emission into a single mode of the radiation field,

- indistinguishable photons with immaculate temporal and spatial coherence, and

- reversible quantum state mapping and entanglement between atoms and photons.

We emphasise that no single-photon source has been realised so far which meets all of the above criteria. For instance, photon sources based on spontaneous parametric down conversion are inherently probabilistic, and cannot reach the desired degree of determinism, even when assisted by an heralding signal. For other sources that exploit the spontaneous emission of single photons into freespace, from emitters like trapped atoms, ions, molecules, or quantum defects in solid-state systems, the obvious lack of directionality can be overcome by collecting photons from a large solid angle. Results on entangling atoms or ions with emitted photons have been obtained $[3,4]$, as well as teleportation of quantum states [5]. However, as the restriction of the solid angle diminishes the efficiency, these sources are rendered probabilistic as well.

Instead, with the single emitter being placed inside an optical cavity, the Purcell effect can be utilised, which, together with coherent control of the state dynamics, allows for deterministic generation of single photons. As discovered by Purcell [6], the spatial mode density inside a cavity is altered substantially, such that the spontaneous emission rate can be either enhanced $(f>1)$ or inhibited $(f<1)$ by the Purcell factor

$$
f=\frac{3 Q \lambda^{3}}{4 \pi^{2} V}
$$

depending on the cavity's mode volume, $V$, and quality factor, $Q$. More importantly, the probability of spontaneous emission into the cavity is given by $\beta=f /(f+1)$. The field modes in the cavity are quantised, and with a suitable spacing between cavity modes, the emitter only couples to a single field mode. Provided the mode volume of the cavity is sufficiently small, the emitter and cavity couple so strongly that the emission probability into the cavity, $\beta$, is significantly larger than the probability for spontaneous emission into free space. Hence, in principle, a deterministic photon emission into a single field mode can be achieved with an efficiency close to unity for very large $f$. These effects have first been observed by Carmichael et al. [7] and De Martini et al. [8]. Moreover, with the coherence properties uniquely determined by the parameters of the cavity and the driving process, one should be able to obtain indistinguishable photons from different cavities. Note also that state mapping and entanglement between atomic spin and photon polarisation has recently been demonstrated in cavity-based singlephoton emitters $[9,10]$. Additionally the reversibility of the photon generation process, and quantum networking between different cavities has been predicted [11, 12], and demonstrated [13].

In the next section, we thoroughly discuss the elementary principles of cavity-QED that apply whenever a single quantum system is coupled to a quantised field mode of a cavity. We then show in section 3 how to exploit these effects to generate single photons on demand in the strong coupling regime and the bad cavity limit, using either an adiabatic driving technique or a sudden excitation of the emitter. In section 4 , we elucidate how to study the properties of the photons: their singleness and their temporal and spectral characteristics. In particular, we focus on the first and second order coherence properties of the light. The section explains how to analyse and measure the photon counting statistics using a HanburyBrown \& Twiss interferometer to determine whether the photons arrive one-by-one, and how to obtain information on their mutual bandwidth and/or temporal jitter from two-photon interference experiments of the HongOu-Mandel type. In section 5, we introduce a classification scheme for tracking the performance and properties of cavity-based single-photon emitters. This scheme allows us to assess the advantages and disadvantages of the most significant approaches, in particular with regard to photonic quantum computing, such as linear optical quantum computing (LOQC) [2] and quantum communication [14], the two most promising applications to date. We also discuss the most prominent experimental achievements and examine the different approaches for obtaining single photons from cavities using either atoms, ions or quantum dots as photon emitters.

\section{ATOM-PHOTON INTERACTION IN RESONATORS}

In this section we discuss how a single quantum system, which shows discrete energy levels like an individual atom or ion, couples to a quantised mode of the radiation field. We first introduce the relevant features of cavity-QED and the Jaynes-Cummings model [15, 16], after which we focus on three-level atoms with two dipole transitions driven by two radiation fields. One of the fields is from a laser, the other is the cavity field strongly coupled to the atom[115]. Furthermore, we shortly explain how the behaviour of a coupled-atom system depends on the most relevant cavity parameters, such as the cavity's mode volume and its finesse.

\section{A. Field quantisation in cavity QED}

Let us consider the coupling of a discrete quantum system to a Fabry-Perot cavity with mirror separation $l$ and reflectivity $\mathcal{R}$. The cavity has a free spectral range $\Delta \omega_{F S R}=2 \pi \times c /(2 l)$, and its finesse is defined as $\mathcal{F}=\pi \sqrt{\mathcal{R}} /(1-\mathcal{R})$. In the vicinity of a resonance, the transmission profile is Lorentzian with a linewidth 
(FWHM) of $2 \kappa=\Delta \omega_{F S R} / \mathcal{F}$, which is twice the decay rate, $\kappa$, of the cavity field. Curved mirrors are often used to restrict the cavity eigenmodes to geometrically stable Hermite-Gaussian or Laguerre-Gaussian modes. We assume the atom is coupled to just one of these modes, characterised by its mode function $\psi_{C}(\mathbf{r})$ and its resonance frequency $\omega_{C}$. In general, the state vector is a superposition of photon-number states, $|n\rangle$, and for $n$ photons in the mode the total energy is $\hbar \omega_{C}\left(n+\frac{1}{2}\right)$. The equidistant energy spacing imposes an analogue treatment of the cavity as an harmonic oscillator. Consequently, creation and annihilation operators for a photon, $\hat{a}^{\dagger}$ and $\hat{a}$, are used to express the Hamiltonian of the cavity,

$$
H_{C}=\hbar \omega_{C}\left(\hat{a}^{\dagger} \hat{a}+\frac{1}{2}\right) .
$$

Note that this Hamiltonian does not take account of any losses. In a real cavity, all photon number states decay until thermal equilibrium with the environment is reached. In the optical domain, the latter corresponds to the vacuum state, $|0\rangle$, with no photons remaining in the cavity.

\section{B. Two-level atom}

We now analyse how the cavity field interacts with a two-level atom with ground state $|g\rangle$ and excited state $|x\rangle$ of energies $\hbar \omega_{g}$ and $\hbar \omega_{x}$, respectively, and transition dipole moment $\mu_{x g}$. The Hamiltonian of the atom reads

$$
H_{A}=\hbar \omega_{g}|g\rangle\left\langle g\left|+\hbar \omega_{x}\right| x\right\rangle\langle x| .
$$

The coupling to the field mode of the cavity is expressed by the atom-cavity coupling constant,

$$
g(\mathbf{r})=g_{0} \psi_{C}(\mathbf{r}), \text { with } g_{0}=\sqrt{\left(\mu_{x g}^{2} \omega_{C}\right) /\left(2 \hbar \epsilon_{0} V\right)},
$$

where $V$ is the mode volume of the cavity. As the atom is barely moving during the interaction, we can safely disregard its external degrees of freedom. Furthermore we assume maximum coupling, i.e. $\psi_{C}\left(\mathbf{r}_{\text {atom }}\right)=1$, so that one obtains $g(\mathbf{r})=g_{0}$. In a closed system, any change of the atom's internal state must be reflected by a corresponding change of the cavity's photon number, $n$. Hence the interaction Hamiltonian of the atom-cavity system reads

$$
H_{\text {int }}=-\hbar g_{0}\left[|x\rangle\left\langle g\left|\hat{a}+\hat{a}^{\dagger}\right| g\right\rangle\langle x|\right] .
$$

For a given excitation number $n$, only the product states $|g, n\rangle$ and $|x, n-1\rangle$ are coupled. If the cavity is resonant with the atomic transition, the population oscillates with the Rabi frequency $\Omega_{C}=2 g_{0} \sqrt{n}$ between these states.

The eigenfrequencies of the total Hamiltonian, $H=$ $H_{C}+H_{A}+H_{\text {int }}$, can be found easily. In the rotating wave approximation, they read

$$
\omega_{n}^{ \pm}=\omega_{C}\left(n+\frac{1}{2}\right)+\frac{1}{2}\left(\Delta_{C} \pm \sqrt{4 n g_{0}^{2}+\Delta_{C}^{2}}\right),
$$

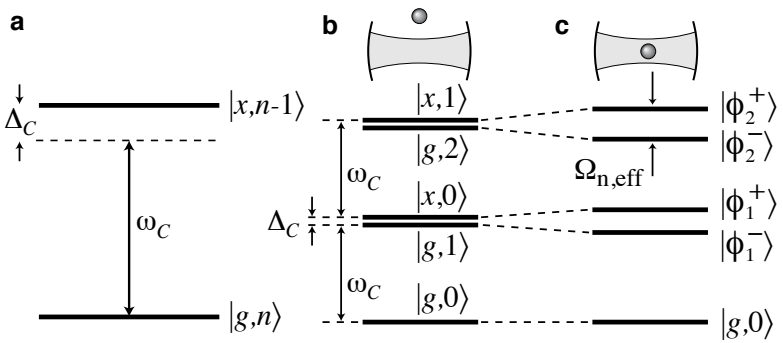

FIG. 1: (a) A two-level atom with ground state $|g\rangle$ and excited state $|x\rangle$ coupled to a cavity containing $n$ photons. In the dressed-level scheme of the combined atom-cavity system with the atom outside (b) or inside (c) the cavity, the state doublets are either split by $\Delta_{C}$ or by the effective Rabi frequency, $\Omega_{\mathrm{n}, \mathrm{eff}}$, respectively.
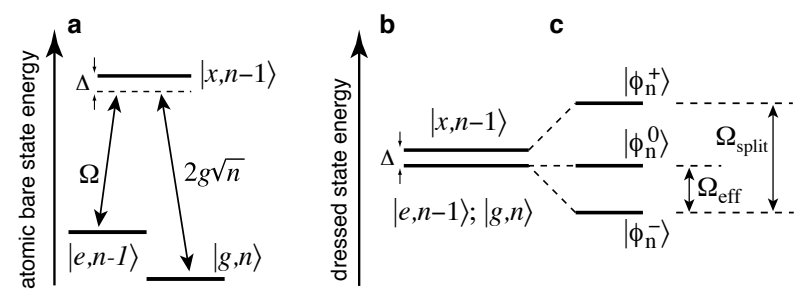

FIG. 2: (a) A three-level atom driven by a classical laser field of Rabi frequency $\Omega$, coupled to a cavity containing $n$ photons. (b) Dressed-level scheme of the combined system without coupling, and (c) for an atom interacting with laser and cavity. The triplet is split by $\Omega_{\text {split }}=\sqrt{4 n g_{0}^{2}+\Omega^{2}+\Delta^{2}}$. In the limit of a large detuning $\Delta$, the Raman transition $\mid e, n-$ $1\rangle \leftrightarrow|g, n\rangle$ is driven at the effective Rabi frequency $\Omega_{\mathrm{eff}}=$ $\frac{1}{2}\left(\Omega_{\text {split }}-|\Delta|\right) \approx\left(4 n g_{0}^{2}+\Omega^{2}\right) /|4 \Delta|$.

where $\Delta_{C}=\omega_{x}-\omega_{g}-\omega_{C}$ is the detuning between atom and cavity. The level splitting between the two corresponding eigenstates, $\Omega_{\mathrm{n} \text {,eff }}=\sqrt{4 n g_{0}^{2}+\Delta_{C}^{2}}$, is the effective Rabi frequency at which the population oscillates between states $|g, n\rangle$ and $|x, n-1\rangle$. This means that the cavity field stimulates the emission of an excited atom into the cavity, thus de-exciting the atom and increasing the photon number by one. Subsequently, the atom is re-excited by absorbing a photon from the cavity field, and so forth. In particular, an excited atom and a cavity containing no photon are sufficient to start the oscillation between $|x, 0\rangle$ and $|g, 1\rangle$ at frequency $\sqrt{4 g_{0}^{2}+\Delta_{C}^{2}}$. This phenomenon is known as vacuum-Rabi oscillation, and for $\Delta_{C}=0$, the resulting oscillation frequency $2 g_{0}$ is called vacuum-Rabi frequency.

To summarise, the atom-cavity interaction splits the photon number states into doublets of non-degenerate dressed states, which are named after Jaynes and Cummings $[15,16]$. Only the ground state $|g, 0\rangle$ is not coupled to any other state and is therefore not subject to any energy shift or splitting. 


\section{Three-level atom}

Now we consider an atom with a $\Lambda$-type three-level scheme providing transition frequencies $\omega_{x e}=\omega_{x}-\omega_{e}$ and $\omega_{x g}=\omega_{x}-\omega_{g}$ as depicted in fig. 2. The $|e\rangle \leftrightarrow|x\rangle$ transition is driven by a classical light field of frequency $\omega_{L}$ with Rabi frequency $\Omega$, and a cavity mode with frequency $\omega_{C}$ couples to the $|g\rangle \leftrightarrow|x\rangle$ transition. If we define the respective detunings as $\Delta_{L}=\omega_{x e}-\omega_{L}$ and $\Delta_{C}=\omega_{x g}-\omega_{C}$, and assume that the driving laser and the cavity only couple to their respective transitions, the behaviour of the atom-cavity system is described by the interaction Hamiltonian

$$
\begin{aligned}
H_{\mathrm{int}}=\hbar[ & \Delta_{L}|e\rangle\left\langle e\left|+\Delta_{C}\right| g\right\rangle\langle g|-\frac{\Omega}{2}(|x\rangle\langle e|+| e\rangle\langle x|) \\
& \left.-g_{0}\left(|x\rangle\left\langle g\left|a+a^{\dagger}\right| g\right\rangle\langle x|\right)\right] .
\end{aligned}
$$

Given an arbitrary excitation number $n$, this Hamiltonian couples only the three states $|e, n-1\rangle,|x, n-1\rangle$, $|g, n\rangle$. For this triplet and a Raman-resonant interaction with $\Delta_{L}=\Delta_{C} \equiv \Delta$, the eigenfrequencies of the coupled system read

$$
\begin{aligned}
& \omega_{n}^{0}=\omega_{C}\left(n+\frac{1}{2}\right) \quad \text { and } \\
& \omega_{n}^{ \pm}=\omega_{C}\left(n+\frac{1}{2}\right)+\frac{1}{2}\left(\Delta \pm \sqrt{4 n g_{0}^{2}+\Omega^{2}+\Delta^{2}}\right)
\end{aligned}
$$

The Jaynes-Cummings doublets of the two-level atom are now replaced by triplets,

$$
\begin{aligned}
\left|\phi_{n}^{0}\right\rangle= & \cos \Theta|e, n-1\rangle-\sin \Theta|g, n\rangle, \\
\left|\phi_{n}^{+}\right\rangle= & \cos \Phi \sin \Theta|e, n-1\rangle-\sin \Phi|x, n-1\rangle \\
& +\cos \Phi \cos \Theta|g, n\rangle, \\
\left|\phi_{n}^{-}\right\rangle= & \sin \Phi \sin \Theta|e, n-1\rangle+\cos \Phi|x, n-1\rangle \\
& +\sin \Phi \cos \Theta|g, n\rangle,
\end{aligned}
$$

where the mixing angles $\Theta$ and $\Phi$ are given by

$$
\tan \Theta=\frac{\Omega}{2 g_{0} \sqrt{n}}, \quad \tan \Phi=\frac{\sqrt{4 n g_{0}^{2}+\Omega^{2}}}{\sqrt{4 n g_{0}^{2}+\Omega^{2}+\Delta^{2}}-\Delta} .
$$

We note that the interaction with the light lifts the degeneracy of the three eigenstates as soon as the Rabi frequencies are non-zero. Furthermore, we emphasise that neither $\left|\phi_{n}^{0}\right\rangle$ is subject to an energy shift, nor does the excited atomic state contribute to it. It is therefore often called a 'dark state' since it cannot decay by spontaneous emission.

In the limit of vanishing $\Omega$, the states $\left|\phi_{n}^{ \pm}\right\rangle$correspond to the Jaynes-Cummings doublet and the third eigenstate, $\left|\phi_{n}^{0}\right\rangle$, coincides with $|e, n-1\rangle$. Note that $\omega_{n}^{0}$ is not affected by $\Omega$ or $g_{0}$. Therefore transitions between the dark states $\left|\phi_{n+1}^{0}\right\rangle$ and $\left|\phi_{n}^{0}\right\rangle$ are always in resonance with the cavity. This holds, in particular, for the transition from $\left|\phi_{1}^{0}\right\rangle$ to $\left|\phi_{0}^{0}\right\rangle \equiv|g, 0\rangle$ since the $n=0$ state does not split (the corresponding states $|e,-1\rangle$ and $|x,-1\rangle$ do not exist).

\section{Cavity-coupling regimes}

In the preceding sections, we have been considering the interaction Hamiltonian and the associated eigenvalues and dressed eigenstates that one obtains whenever a two- or three-level quantum system is coupled to a cavity. We have been neglecting the transverse polarisation decay rate, $\gamma_{\perp}$, of the quantum system, and also the field-decay rate of the cavity, $\kappa$, has not been taken into account[116]. It is evident that both relaxation rates result in a damping of a possible vacuum-Rabi oscillation between states $|x, 0\rangle$ and $|g, 1\rangle$, and therefore determine how the atom is coupled to the cavity. Two extreme cases are worth mentioning here, namely the

- Strong-coupling regime, with $g_{0} \gg\left\{\kappa, \gamma_{\perp}\right\}$. In this case, the damping rates have a negligible effect on the time evolution of the coupled atom-cavity system, and vacuum-Rabi oscillations can occur. For a three-level atom exposed to driving laser and cavity, the condition sometimes reduces to $g_{0} \gg \kappa$, provided one can eliminate the excited atomic state from the description.

- Bad-cavity regime, with $\kappa \gg g_{0}^{2} / \kappa \gg \gamma_{\perp}$. This results in a strong damping and quasi-stationary quantum states of the coupled system (see section III B).

Two properties of the cavity can be used to distinguish between these regimes: First the strength of the atomcavity coupling, $g_{0} \propto 1 / \sqrt{V} \propto l^{-3 / 4}$ (dependant upon the dimensions of the cavity mode, with the transverse mode area $\pi w_{0}^{2} \propto \sqrt{l}$ for near-planar cavities), and second the finesse $\mathcal{F}$ of the resonator, which depends on the mirror reflectivity. The finesse corresponds to the average number of round trips in the cavity before a photon gets lost by transmission through one of the cavity mirrors, and can also be expressed as the ratio between free spectral range $\Delta \omega_{F S R}$ and cavity linewidth $2 \kappa$. Consequently a trade-off between $g_{0}$ and $\kappa$ is needed to keep one high and the other low, as the latter increases with $1 /(\mathcal{F} \times l)$. Fig. 3 shows the two coupling regimes as a function of finesse and cavity length. For the strong-coupling regime that relies on a high value of $g_{0}$ and therefore a short cavity of small mode volume, only a cavity of sufficiently high finesse will keep $\kappa$ small enough. This usually requires a mirror reflectivity $R \geq 99.999 \%$.

\section{SINGLE-PHOTON EMISSION}

In this section, we introduce several different ways of producing single photons from a coupled atom-cavity system. This encompasses cavity-enhanced spontaneous emission and Raman transitions stimulated by the vacuum field while driven by classical laser pulses. In particular, we introduce a scheme for adiabatic coupling between a single atom and an optical cavity, which is based 


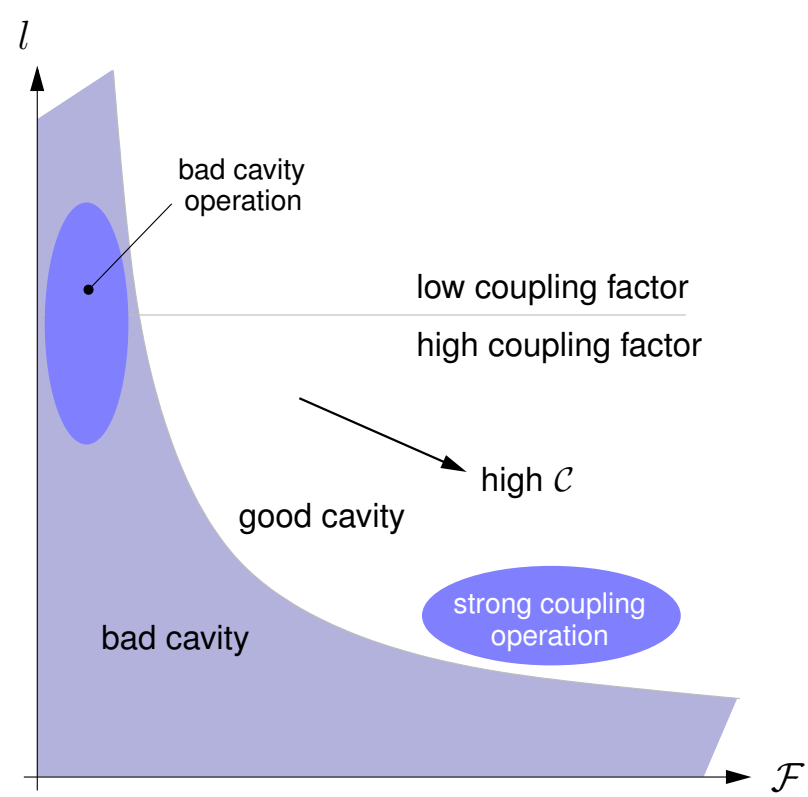

FIG. 3: Cavity coupling regimes as determined by cavity finesse, $\mathcal{F}$, and cavity length, $l$. $\mathcal{C}$ is the cooperativity parameter.

on a unitary evolution of the coupled atom-cavity system [17], and is therefore intrinsically reversible. This scheme either allows one to populate photon-number states on demand in the cavity, or to emit single optical photons into a well-defined mode of the radiation field outside the cavity.

For a photon emission from the cavity to take place, it is evident that a finite value of $\kappa$ is mandatory, otherwise any light would remain trapped in the cavity. Moreover, as $\kappa$ is the decay rate of the cavity field, the associated minimum length of an emitted photon is in most cases given by $\kappa^{-1}$. We also emphasise that $\gamma_{\perp}$ plays a crucial role in most experimental settings, since it accounts for the spontaneous emission into non-cavity modes, and therefore leads to a reduction of efficiency. The relation of the atom-cavity coupling constant and the Rabi frequency of the driving field to the two decay rates can be used for marking the difference between three basic classes of single-photon emission schemes from cavityQED systems:

I. Cavity-enhanced spontaneous emission: A sudden excitation process (e.g. a short $\pi$ pulse with $\Omega \gg\left\{g_{0}, \kappa, \gamma_{\perp}\right\}$, or some internal relaxation cascade starting from an energetically higher state) drives the quantum system into its excited state. Subsequently, a photon gets spontaneously emitted either into the cavity or into free space. Due to the Purcell effect, the emission probability into the cavity is strongly enhanced by the Purcell factor $f=2 C$, where $C$ is the cooperativity parameter of the cavity.

II. Steady-state evolution in the bad-cavity regime: A three-level system is coupled to the cavity and a driving laser. In the bad-cavity regime, the cavity decay $\kappa$ is stronger than $g_{0}$ and $\Omega$. Therefore the system is overdamped to a quasi-stationary state at any time of the interaction. As the stationary state of the coupled system depends on $\Omega(t)$, the envelope function of the emitted photon can be controlled to a large extend. However, with some transient population placed in the excited state, one cannot rule out spontaneous emission losses into non-cavity modes and the efficiency is reduced.

III. Dynamic evolution in the strong-coupling regime: A three-level system is strongly coupled to the cavity and a driving laser, with $\left\{\Omega, g_{0}\right\} \gg \kappa$. Therefore the cavity decay may be neglected on the time scale of the excitation, and the coupled system undergoes stimulated Raman transitions between the two atomic ground states and the associated zero- and one-photon states of the cavity. For instance, the driving process can be implemented in form of an adiabatic passage (STIRAP process [17]) or a far-off resonant Raman process to avoid placing any transient population into the excited level, thus reducing losses due to spontaneous emission into free space. In that case, an efficiency for photon generation close to unity can be reached. Once a photon is placed into the cavity, it gets emitted due to the finite cavity-decay rate $\kappa$.

We emphasise that these three scenarios represent extreme cases. Any real cavity-based single-photon emitter combines several of the above aspects, according to the parameters of the coupled system and the specific characteristics of the driving field. The following three sections discuss the three different approaches in more detail.

\section{A. Cavity-enhanced spontaneous emission}

As a starting point, we assume that some excitation process (e.g. a short $\pi$ pulse with $\Omega \gg\left\{g_{0}, \kappa, \gamma_{\perp}\right\}$, or some internal relaxation cascade starting from an energetically higher state) has been implemented to drive the quantum system suddenly into its excited state $|x, 0\rangle$. Hence we are free to treat the system as a simple excited two-level quantum system coupled to an empty optical cavity. This particular situation is the text book example of cavity-QED that has been thoroughly analysed in the past. In fact, it has been proposed by Purcell [6] and demonstrated by Heinzen et al. [18] and Morin et al. [19] that the spontaneous emission properties of an atom coupled to a cavity are significantly different from those in free space. For an analysis of the atom's behaviour, it suffices to look at the evolution of $n=1$ doublet under the influence of the atomic polarisation decay rate $\gamma_{\perp}$ and the cavity-field decay rate $\kappa$. As non-cavity spontaneous decay of the atom and photon emission through one of the cavity mirrors both lead the system into state 
$|g, 0\rangle$, i.e. a dressed state outside the $n=1$ doublet, we can deal with these decay processes phenomenologically by introducing non-hermitian terms into the interaction Hamiltonian,

$$
H_{\text {int }}^{\prime}=-\hbar g_{0}\left(|x\rangle\left\langle g\left|\hat{a}+\hat{a}^{\dagger}\right| g\right\rangle\langle x|\right)-i \hbar \gamma_{\perp}|x\rangle\langle x|-i \hbar \kappa \hat{a}^{\dagger} \hat{a} .
$$

Figure 4(a) shows the time evolution of the atom-cavity system when $\kappa>g_{0}$. The strong damping of the cavity's one-photon state inhibits any vacuum-Rabi oscillation, since the photon is emitted from the cavity before it can be reabsorbed by the atom. Therefore the transient population in state $|g, 1\rangle$ is negligible if the atom is initially in its excited state $|x\rangle$. In this case, the adiabatic approximation $\dot{c}_{g} \approx 0$ can be applied, which gives

$$
\frac{d}{d t} c_{x}=-\gamma_{\perp} c_{x}-\frac{g_{0}^{2}}{\kappa} c_{x}
$$

with the solution

$$
c_{x}(t)=\exp \left(-\left[\gamma_{\perp}+\frac{g_{0}^{2}}{\kappa}\right] t\right) .
$$

It is straightforward to see that the ratio of the emission rate into the cavity, $g_{0}^{2} / \kappa$, to the spontaneous emission probability into free space becomes $g_{0}^{2} /\left(\kappa \gamma_{\perp}\right) \equiv f$, i.e. the Purcell factor. It equals twice the one-atom cooperativity parameter, $C$, originally introduced in the context of optical bistability [20]. Hence the photon-emission probability from the cavity reads $P_{\text {Emit }}=2 C /(2 C+1)$. Note that the atom radiates mainly into the cavity if $g_{0}^{2} / \kappa \gg \gamma_{\perp}$. Together with $\kappa \gg g_{0}$, this condition constitutes the bad-cavity regime.

The other extreme we are considering is the strongcoupling regime, $g_{0} \gg\left(\kappa, \gamma_{\perp}\right)$. In this case the atomcavity system is subject to vacuum-Rabi oscillations between $|x, 0\rangle$ and $|g, 1\rangle$, with both states decaying at the respective rates $\gamma_{\perp}$ and $\kappa$. Figure $4(\mathrm{~b})$ shows a situation where the atom-cavity coupling, $g_{0}$, saturates the $|x, 0\rangle \leftrightarrow|g, 1\rangle$ transition. On average, the probabilities to find the system in either one of these two states are equal, and therefore the average ratio of the emission probability into the cavity to the spontaneous emission probability into free space is given by $\kappa / \gamma_{\perp}$. The vacuumRabi oscillation in this particular case gives rise to a pronounced amplitude modulation of the photons emitted from the cavity, so that their coherence properties are not as favourable as in the other schemes discussed here.

We emphasise that the enhancement of the atom's spontaneous emission into the cavity cannot be denoted in terms of simple analytic expressions in intermediate coupling regimes. Nonetheless, the emission probability into the cavity will be enhanced as long as the cavity mode is resonant with the atomic transition.

\section{B. Steady-state evolution in the bad-cavity regime}

To take the effect of the excitation process into account, we now consider a $\Lambda$-type three-level atom coupled
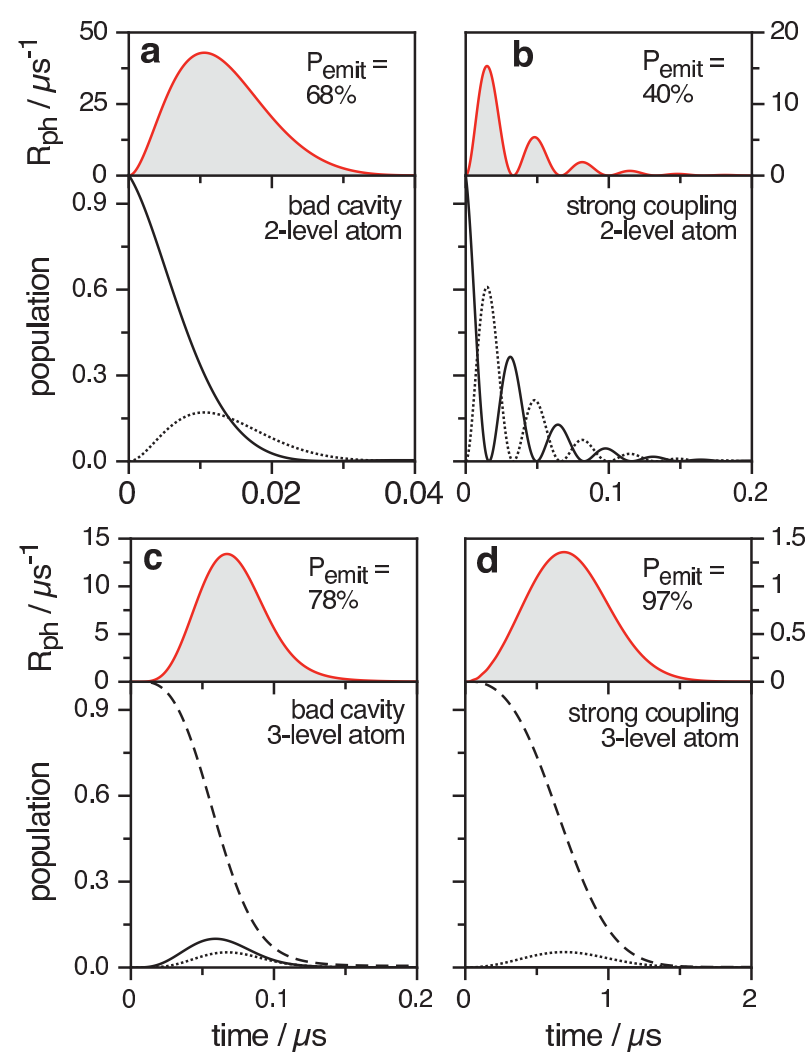

FIG. 4: Time evolution of the system and photon emission rate $R_{p h}=2 \kappa \rho_{g g}$ for different coupling cases. In the upper row, an excited two-level atom is coupled to the cavity and populations $\rho_{x x}$ (solid line) and $\rho_{g g}$ (dotted line) of the product states $|x, 0\rangle$ and $|g, 1\rangle$ are shown. In the lower row, a three-level atom-cavity system prepared in $|e, 0\rangle$ is exposed to a pump pulse driving $|e\rangle-|x\rangle$ while the cavity couples $|x\rangle$ and $|g\rangle$. The population $\rho_{e e}$ of the initial state is shown as a dashed line. The left column displays the bad-cavity regime with $\left(g_{0}, \gamma_{\perp}, \kappa\right)=$ $2 \pi \times(15,3,20) \mathrm{MHz}$, and the right column the strong-coupling regime with $\left(g_{0}, \gamma_{\perp}, \kappa\right)=2 \pi \times(15,3,2) \mathrm{MHz}$. The pump pulses read $\Omega(t)=g_{0} \sin (\pi t / 200 n s)$ in (c), and $\Omega(t)=$ $g_{0} \times t / 1 \mu \mathrm{s}$ in (d). No transient population is found in $\rho_{x x}$ in the latter case. In all cases, the overall photon-emission probability is given by $P_{\text {Emit }}=\int R_{p h} d t$.

to a cavity. We first follow Law et al. [21, 22] and consider the bad-cavity regime, $\kappa \gg g_{0}^{2} / \kappa \gg \gamma_{\perp}$, where the loss of excitation into unwanted modes of the radiation field is small. We assume that the atom's $|e\rangle-|x\rangle$ transition is excited by a pump pulse while the atom emits a photon into the cavity by enhanced spontaneous emission. In this regime, the cavity-field decay rate $\kappa$ sets the fastest time scale, while the spontaneous emission rate into the cavity, $g_{0}^{2} / \kappa$, dominates the incoherent decay of the polarisation from the excited atomic state. Assuming that any decay leads to a loss from the three-level system, the evolution of the wave vector is governed by the non-Hermitian Hamiltonian

$$
H_{i n t}^{\prime}=H_{i n t}-i \hbar \kappa \hat{a}^{\dagger} \hat{a}-i \hbar \gamma_{\perp}|x\rangle\langle x|,
$$


with $H_{\text {int }}$ given in (6). To simplify the analysis, we consider only the vacuum state, $|0\rangle$, and the one-photon state, $|1\rangle$, of the cavity. Hence the state vector can be written as

$$
|\Psi(t)\rangle=c_{e}(t)|e, 0\rangle+c_{x}(t)|x, 0\rangle+c_{g}(t)|g, 1\rangle,
$$

where $c_{e}, c_{x}$ and $c_{g}$ are complex amplitudes. The time evolution of the amplitudes is given by the Schrödinger equation, $i \hbar \frac{d}{d t}|\Psi\rangle=H_{\text {int }}^{\prime}|\Psi\rangle$, which yields

$$
\begin{aligned}
i \dot{c}_{e} & =\frac{1}{2} \Omega(t) c_{x} \\
i \dot{c}_{x} & =\frac{1}{2} \Omega(t) c_{e}+g_{0} c_{g}-i \gamma_{\perp} c_{x} \\
i \dot{c}_{g} & =g_{0} c_{x}-i \kappa c_{g},
\end{aligned}
$$

with the initial condition $c_{e}(0)=1, c_{x}(0)=c_{g}(0)=0$ and $\Omega(0)=0$. An adiabatic solution of $(15)$ is found as long as $\Omega(t) \ll g_{0}^{2} / \kappa$, as the decay is so fast that $c_{x}$ and $c_{g}$ are nearly time independent. This allows one to make the approximations $\dot{c}_{x}=0$ and $\dot{c}_{g}=0$, with the result

$$
\begin{aligned}
c_{e}(t) & \approx \exp \left(-\frac{\alpha}{4} \int_{0}^{t} \Omega^{2}\left(t^{\prime}\right) d t^{\prime}\right) \\
c_{x}(t) & \approx-\frac{i}{2} \alpha \Omega(t) c_{e}(t) \\
c_{g}(t) & \approx-\frac{i}{\kappa} g_{0} c_{x}(t),
\end{aligned}
$$

where $\alpha=2 /\left(2 \gamma_{\perp}+2 g_{0}^{2} / \kappa\right)$. Since photon emissions from the cavity only occur if the system is in $|g, 1\rangle$, the photonemission rate reads $R_{p h}(t)=2 \kappa\left|c_{g}(t)\right|^{2}$, thus yielding a photon-emission probability of

$$
\begin{aligned}
P_{\text {Emit }} & =\int R_{p h}(t) d t \\
& =\frac{g_{0}^{2} \alpha}{\kappa}\left[1-\exp \left(-\frac{\alpha}{2} \int \Omega^{2}(t) d t\right)\right] \longrightarrow \frac{g_{0}^{2} \alpha}{\kappa}
\end{aligned}
$$

Note that the exponential in (17) vanishes if the area $\int \Omega(t) d t$ of the exciting pump pulse is large enough. In this limit, the photon-emission probability does not depend on the shape and amplitude of the pump pulse. With a suitable choice of $g_{0}, \alpha$, and $\kappa$, high photonemission probabilities can be reached [22].

\section{Dynamic evolution in the strong-coupling regime}

To study the effect of the exciting laser pulse in the strong-coupling regime, we again consider a $\Lambda$-type threelevel atom coupled to a cavity. We assume that the strong-coupling condition also applies to the Rabi frequency of the driving field, i.e. $\left\{g_{0}, \Omega\right\} \gg\left\{\kappa, \gamma_{\perp}\right\}$. In this case, we can safely neglect the effect of the two damping rates on the time scale of the excitation and look for a method to effectively stimulate a Raman transition between the two ground states, which also places a single photon into the cavity.
The most promising approach to achieve that goal is to implement an adiabatic passage in the optical domain between these two states $[23,24]$. In fact, adiabatic passage methods have been used for coherent population transfer in atoms or molecules for many years. For instance, if a Raman transition is driven by two distinct pulses of variable amplitudes, effects like electromagnetically induced transparency (EIT) [25, 26], slow light [27, 28], and stimulated Raman scattering by adiabatic passage (STIRAP) [17] are observed. All of these effects have been demonstrated with classical light fields, and have the property in common that the system's state vector, $|\Psi\rangle$, always coincides with a single eigenstate, e.g. $\left|\phi_{n}^{0}\right\rangle$, of the time-dependent interaction Hamiltonian. In principle, the time evolution of the system is completely controlled by the variation of this eigenstate. However, a more detailed analysis $[23,29]$ reveals that the eigenstates must change slowly with respect to the eigenfrequency differences. Adiabaticity is assured if the condition $\left|\omega_{n}^{ \pm}-\omega_{n}^{0}\right| \gg\left|\left\langle\phi_{n}^{ \pm}\left|\frac{d}{d t}\right| \phi_{n}^{0}\right\rangle\right|$ is met throughout the interaction, and as long as the system does not decay via some other channel. In this context, the non-decaying dark state, $\left|\phi_{n}^{0}\right\rangle$, is of enormous significance.

It follows that a three-level atom-cavity system, once prepared in $\left|\phi_{n}^{0}\right\rangle$, should stay there, thus allowing one to control the relative population of the contributing product states, $|e, n-1\rangle$ and $|g, n\rangle$, by simply adjusting the pump Rabi frequency, $\Omega$. To show this, let us start with a system initially prepared in state $|e, n-1\rangle$. As can be seen from Eq. (8), this state coincides with $\left|\phi_{n}^{0}\right\rangle$ if the condition $2 g_{0} \sqrt{n} \gg \Omega$ is met in the beginning of the interaction. Once the system has been successfully prepared in the dark state, the ratio between the populations of the contributing states reads

$$
\frac{|\langle e, n-1 \mid \Psi\rangle|^{2}}{|\langle g, n \mid \Psi\rangle|^{2}}=\frac{4 n g_{0}^{2}}{\Omega^{2}} .
$$

As proposed in [30], we now assume that an atom in state $|e\rangle$ is placed in a cavity mode populated with $n-1$ photons driving the $|g, n\rangle \leftrightarrow|x, n-1\rangle$ transition with the effective Rabi frequency $2 g_{0} \sqrt{n}$, such that the initial state coincides with $\left|\phi_{n}^{0}\right\rangle$. The atom is then exposed to a laser pulse coupling the $|e\rangle \leftrightarrow|x\rangle$ transition with a slowly rising amplitude that finally leads to $\Omega \gg 2 g_{0} \sqrt{n}$. The system evolves from $|e, n-1\rangle$ to $|g, n\rangle$, and the photon number increases by one. Without cavity decay, the successive application of this method would allow one to prepare arbitrary photon-number states [31]. To do so, the pump pulse must be turned off suddenly, or the system would adiabatically return to its initial state.

We emphasise that the situation is the same if one starts with an empty cavity, i.e. with the atom-cavity system initially in state $|e, 0\rangle$, application of this method transfers the coupled system into state $|g, 1\rangle$ via the dark state $\left|\phi_{1}^{0}\right\rangle$, with exactly one photon emitted into the cavity mode. In this particular case, the excitation scheme constitutes vacuum-stimulated Raman scattering by adiabatic passage, known as V-STIRAP. In the case of a 
finite cavity decay time $\kappa^{-1}$ much longer than the interaction time, a photon is emitted from the cavity with a probability close to unity and with properties uniquely defined by $\kappa$, after the system has been excited to $|g, 1\rangle$.

In contrast to this idealised scenario, Fig. 4(d) shows a more realistic situation where the photon is generated and emitted from the cavity during the excitation process. This is due to the cavity decay time being comparable or shorter than the exciting laser pulse. Even in this case, no secondary excitations or photon emissions take place, as the system reaches the decoupled state $|g, 0\rangle$ once the photon escapes. However, the photon-emission probability is slightly reduced as the non-Hermitian contribution of $\kappa$ to the interaction Hamiltonian gives rise to a small admixture of $|x, 0\rangle$ to the eigenstate $\left|\phi_{1}^{0}\right\rangle$. The latter is therefore no pure dark state, as it is weakly affected by spontaneous emission losses. Nonetheless, photon-emission probabilities far above $90 \%$ are always attainable if V-STIRAP is applied.

\section{Arbitrary photon shaping}

As we have seen from the previous analysis, the dynamic evolution of the atomic quantum states provides information of the photon emission rate, and thereby also the photon's waveform. This raises the question as to what extent we can arbitrarily shape the photons in time by controlling the driving field's envelope. This is an important problem for applications such as quantum state mapping, where the availability of photon wave packets that are symmetric in space and time allow for a time-reversal of the emission process [11]. Custom photon shaping is also of interest for generating approximate Gaussian pulse shapes which are shown to maximise the tolerance against mode-mismatch in interference-based quantum information processing schemes [32]. Employing photons of soliton-shape for dispersion-free propagation in optical fibres could also help boost quantum communication protocols.

The problem of photon shaping have been addressed traditionally by solving the Master equation of the atomphoton system, which yields the time-dependent probability amplitudes, and by consequence also the wave function of the photon emitted from the cavity [33, 34]. It has recently been shown [35] that this analysis can also be reversed, giving a unambiguous analytic expression for the time evolution of the driving field as a function of the desired shape of the photon. This model is not only valid for V-STIRAP in the strong-coupling and bad-cavity regime, but it generally allows control of the coherence and population flow in any Raman process. Fig. 5 compares some of the results obtained.

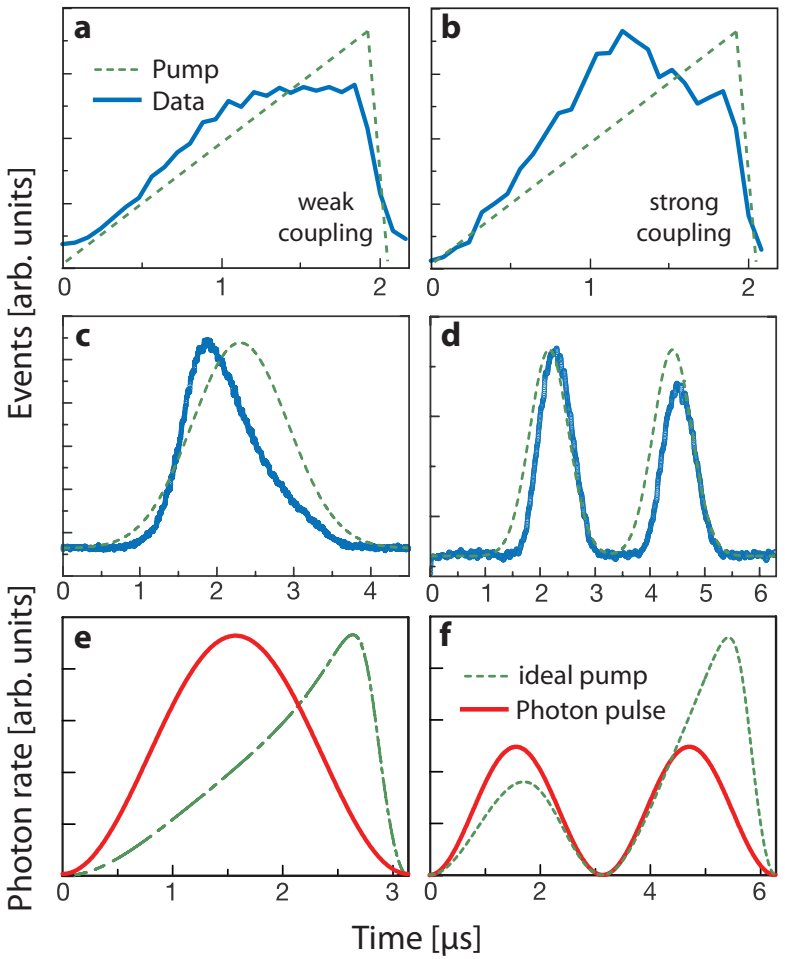

FIG. 5: Shaping the photon. (a-d) show photon shapes realised in several experiments together with the respective driving laser pulses. To obtain this data, the histogram of the photon-detection time has been recorded using several hundred single-photon emissions. The data shown in $(\mathbf{a}+\mathbf{b})$ is taken from [33], with neutral atoms falling through a highfinesse cavity acting as photon emitters. The linear increase in Rabi frequency is the same in both cases, and the difference in photon shape is caused by variations in the coupling strength to the cavity. The data shown in $(\mathbf{c}+\mathbf{d})$ is taken from [34], with a single ion trapped between the cavity mirrors. It impressively shows that the photon shape depends strongly on the driving laser pulse. (Reprinted by permission from Macmillan Publishers Ltd: Nature (M. Keller et al. [34]), copyright 2004.) The last row (e+f) shows the Rabi frequency one needs to apply to achieve symmetric single or twin-peak photon pulses with an efficiency close to unity. This is a result from an analytic solution of the problem discussed in [35].

\section{SINGLE-PHOTON CHARACTERISATION}

In this section, we elucidate how the properties of photons, namely their singleness, their temporal and their spectral characteristics can be studied. In particular, we focus on the first- and second-order coherence properties of the light intensity. The characterisation of a singlephoton source usually starts with an investigation of the photon statistics, where the observation of antibunching indicates that the source tends to emit single photons. First, we explain how to measure and analyse the photon counting statistics, and how the photon statistics are affected by several properties of the photon emitter, such as 
low efficiency, blinking and intermittent operation, background noise, and a spurious multi-photon contribution. Second, we focus on the coherence and temporal properties of the emitted photons, and show how to obtain information on their mutual bandwidth and/or temporal jitter from two-photon interference experiments.

\section{A. Statistics: photons one-by-one}

The most crucial test for a single-photon emitter consists of a statistical analysis of the emitted photon stream to determine whether the photons arrive one-by-one. For a deterministic source that emits single photons in response to some trigger pulse, a direct measurement of the photon number statistics upon each trigger event would completely characterise the singleness. Ideally, one expects to find probabilities of $P(n=1)=1$ and $P(n \neq 1)=0$. However, as the efficiency of most sources is far below one, it is generally more suitable to verify the sub-Poissonian statistics of the radiation by measuring the second-order intensity correlation function, $g^{(2)}(\tau)$, of the photon stream with a Hanbury-Brown \& Twiss setup [36].

In simple terms, it is needed that the probability of finding another photon is vanishingly small once a first photon has been detected. In principle, this could be accomplished by an ideal photon counter that registers the arrival times of all impinging photons. However, due to the limited dead time of real detectors and their inherent incapability of distinguishing between multiple photon events, a pair of detectors is normally used. HanburyBrown \& Twiss' method works by splitting the incoming photon stream into two using a beamsplitter, after which a measurement of the cross-correlation using two detectors $\left(D_{1}\right.$ and $\left.D_{2}\right)$ gives a signal proportional to the autocorrelation of the light. The principle is depicted in Fig. 6. In the limit of infinitely short detector dead-times,
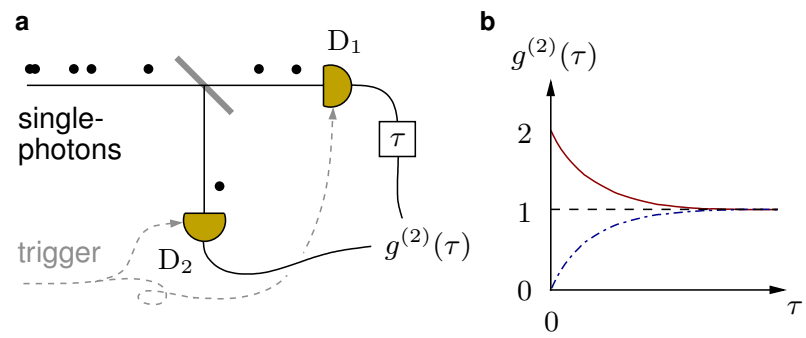

FIG. 6: a) Basic principle of Hanbury-Brown \& Twiss' technique to obtain correlation statistics for single-photon characterisation. The autocorrelation function of the photon stream is measured using two detectors behind a beamsplitter with one signal delayed by $\tau$. b) The correlation function can show signs of either systematic correlations (solid line), random correlations (dashed line), or no correlations (dash-dotted line), where the latter is an indication of non-classical light being measured.

the full autocorrelation function is found as a function of the time delay $\tau$ between photo detections. In terms of the intensity of the light field the autocorrelation function is given by

$$
g^{(2)}(\tau)=\frac{\left\langle I_{1}(t) I_{2}(t+\tau)\right\rangle}{\left\langle I_{1}(t)\right\rangle\left\langle I_{2}(t)\right\rangle},
$$

where $I_{n}(t)$ is the light power recorded by photodetector $n$. Similarly, in terms of probabilities of photon counts for two single-photon detectors, the autocorrelation function is given by the cross-correlation

$$
g^{(2)}(\tau)=\frac{P_{12}(\tau)}{P_{1}(t) P_{2}(t+\tau)},
$$

where $P_{x}: x \in\{1,2,12\}$ is the probability of a single or joint detection events, as given by the counting rates from the two detectors.

A few interesting cases are shown in Fig. 6 and discussed in Ref. [37]. Classical light fulfils the CauchySchwarz inequality, leading to $g^{(2)}(0) \geq g^{(2)}(\tau \neq 0)$, whereas light that violates this inequality must be described by the laws of quantum physics. First experiments demonstrating antibunching, $g^{(2)}(0)<g^{(2)}(\tau \neq$ 0 ), were performed with a beam of atoms [38]. Limitations imposed by number fluctuations [39] were later eliminated by using a single ion [40], atom [41, 42], molecule [43], quantum dot [44], or color centre [45, 46]. For light with $g^{(2)}(0)=0$, photon number states with $n>1$ are totally absent. Hence the value at $\tau=0$ indicates how the expectation value of the photon number is changed upon a first detection event. Classical (thermal) bunching, $g^{(2)}(0)>g^{(2)}(\tau \neq 0)$, has been observed in the fluorescence of a large number of independently radiating atoms as early as 1956 [36], and has regained new interest in the context of cold-atom physics [47, 48]. Only recently, one of has been observing a smooth transition between these two regimes in a cavity-QED experiment [49], with the photon statistics changing from antibunching to bunching as a function of the atom number in the cavity. Note that the special case of a flat correlation function with $g^{(2)}(\tau) \equiv 1$ is found for coherent light, i.e. laser radiation, as its photon statistics is Poissonian. By consequence, the photon statistics is called sub-Poissonian if $g^{(2)}(0)<1$, and super-Poissonian when $g^{(2)}(0)>1$.

In view of the above, $g^{(2)}(0)=1$ implies no change to the mean photon number after detection and corresponds to a completely uncorrelated Poissionian statistics, $g^{(2)}(0)=2$ implies doubling the mean as for thermal statistics, and $g^{(2)}(0)=0$ implies that nothing is left to detect after a first detection, as for the ideal number state $|1\rangle$. In order to quantify the statistics of the photon stream emitted from a given source in terms of its single-photoness, we are therefore generally interested in the autocorrelation value at zero-lag, i.e. when $\tau=0$,

$$
g^{(2)}(0)=\frac{P_{12}}{P_{1} P_{2}} .
$$


Some typical examples of $g^{(2)}(\tau)$ are plotted in Fig. 7 . The graphs are based on experimental data for different cases of continuous versus discrete (pulsed) sources, and for free falling versus trapped atoms. It is seen that the form of $g^{(2)}(\tau)$ essentially resembles that of Fig. 6b for non-classical states, showing clear signs of antibunching as discussed above. Note, however, that traces $7 \mathrm{a}-\mathrm{d}$ seem to indicate super-Poissionian statistics, even after subtraction of the background. This is because the measured photon statistics is additionally affected by the statistical distribution of atoms or ions that are available to emit a photon (or by the intermittent operation in the case of quantum dots, so-called blinking), which adds systematic correlations to the data. In contrast to these, trace $7 \mathrm{e}$ depicts the photon statistics obtained with a single atom trapped inside a cavity. Hence the atom number is not subject to statistical variations and the emitted radiation is sub-Poissonian, as can be seen from the inset. Generally, a Poisson-distributed atom number adds a unit constant to $g^{(2)}\left(\tau<\tau_{c}\right)$, while a thermal distribution adds a constant 2 , and a fixed atom number state $|N\rangle_{e}$ adds a constant $1-1 / N$. In addition, values are also scaled by a factor that is inversely proportional to the average atom number $\bar{N}$ (or to the duty-cycle in case of blinking), which corresponds to an inclusion of dark events and leads to large values of $g^{(2)}\left(\tau_{c}<\tau<\tau_{i}\right)$ for $\bar{N}<1$, where $\tau_{i}$ is the atom-cavity interaction time (or the blinking period). For large delays there are only random correlations remaining, and thus $g^{(2)}(\tau \rightarrow \infty)=1$. The ideal case of a single trapped emitter, $|1\rangle_{e}$, does not affect $g^{(2)}$ for any delay, since $N=\bar{N}=1$. With these effects accounted for, e.g. by using a trigger pulse to exclude dark events and/or conditioning the experiment on the actual presence of a photon emitter in the cavity, the output is normally consistent with the requirements of a singlephoton source, namely to show both sub-Poisson statistics and anti-bunching. For further discussions around this topic we refer to Ref. [50].

\section{B. Indistinguishability: the photon identity}

The requirements on a single-photon source for LOQC and for the entanglement of two distant atoms go far beyond showing sub-Poissonian statistics and antibunching. The realisation of these proposals relies on indistinguishability between the photons, so that even photons from different sources are identical with respect to their frequency, duration and shape. Therefore it is desirable to investigate the spectral and temporal properties of single photons emitted from a given source. A simple correlation measurement between the trigger event and the detection time of the generated photon will reveal the temporal envelope (shape) of the photonic ensemble [33, 34, 52], assuming sufficiently high detector timing resolution. However, as this method is insensitive to the spectral properties of the photons, the measured envelope generally represents temporal multimodes and only
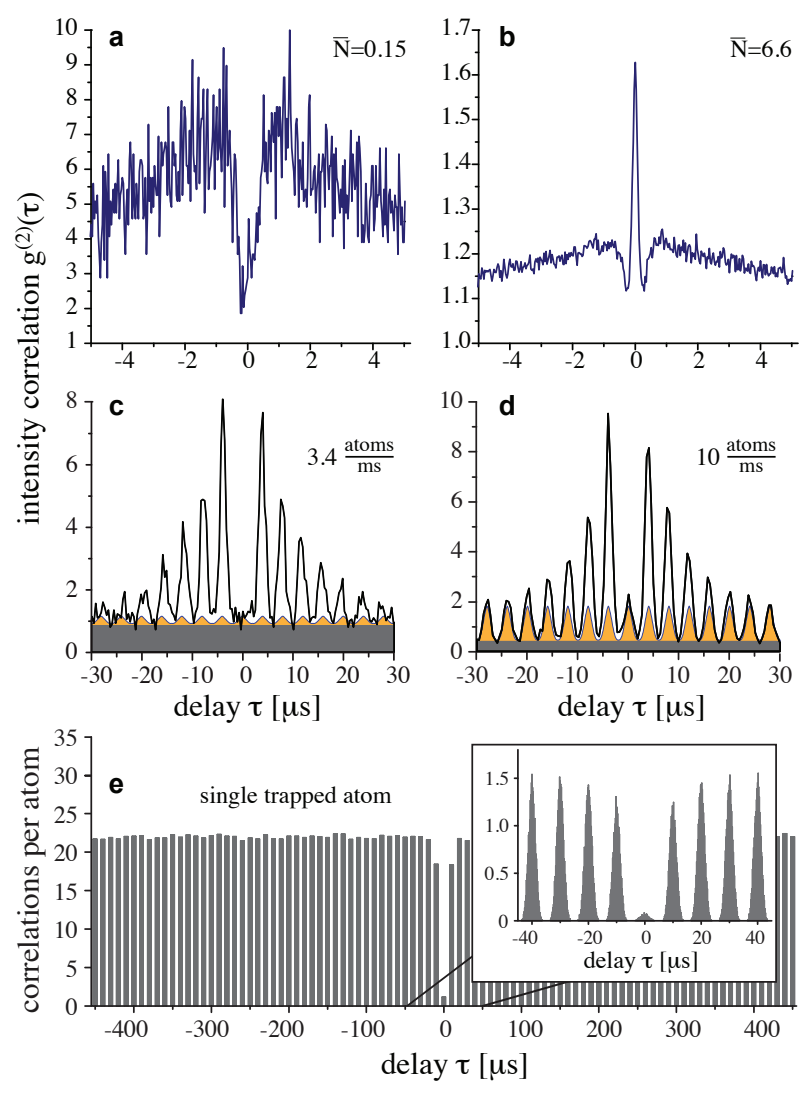

FIG. 7: The intensity correlation function $g^{(2)}(\tau)$ measured using a Hanbury-Brown \& Twiss setup for different types of cavity-based photon emitters. $(\mathbf{a}+\mathbf{b})$ are recorded for a continuously driven system [49], with a stream of atoms falling through the cavity. Antibunching is visible for small average atom number, $\bar{N}$, and a pronounced thermal bunching peak is found for $\bar{N} \gg 1$. (c+d) show the photon statistics for a triggered source, with atoms falling randomly through the cavity $[33,50]$. The Gaussian envelope is due to the limited atom-cavity interaction time, and the comb of peaks reflects the stream of trigger pulses. With the average atom number being small, the missing central peak signals antibunching. Note, however, that the raw data shows no sub-Poissonianess, as it is affected by the random arrival of atoms in the cavity. (e) corresponds to the ideal case, with a single emitter (atom) trapped inside a cavity [51]. Antibunching and subPoissonianess are evident, and no envelope function is visible in the correlation function.

coincides with the single-mode envelope of the photon wavepackets if all photons are identical. A more sophisticated analysis is therefore mandatory.

The standard way to determine the coherence time of a given light source is through measurement of the secondorder interference using a Mach-Zehnder or Michelson interferometer. This measurement can also be done with single photons, so that each single photon follows both paths of the interferometer and interferes with itself. This method has been used to measure the coherence time of solid-state based single-photon sources [53, 54]. However, these experiments cannot reveal jitter in the 
emission time, and they are also not the method of choice for characterising photons of long coherence time, or length, due to the mere impossibility of scanning one interferometer arm by several hundred meters.

In the following, we shortly summarise how to characterise the very narrow frequency bandwidth of single photons created by cavity-based emitters using two-photon interference, avoiding the above restrictions.

\section{Two-photon interference}

The first experiment to investigate the mutual coherence time in photon pairs was performed by Hong et al. [55]. They were measuring the second-order interference between two photons from a parametric down-conversion source by superimposing the signal and the idler photon on a symmetric beamsplitter. The coincidence rate of photodetections at the two output ports of the beamsplitter was measured in dependence of a relative arrivaltime delay between the two photons. Due to their bosonic nature, indistinguishable photons coalescence and leave the beamsplitter as a pair, so that no coincident photo detections are recorded in the two output ports, as described in Fig. 8. If the photons differ slightly, e.g. because they arrive at different times, coincidences will be present. Furthermore, the coincidence rate shows a minimum as a function of the arrival-time delay. For indistinguishable photons, the width of this dip equals the photon coherence time, i.e. duration. Any distinguishability between the interfering photons reduces the dip's depth. However, this method is also problematic with long coherence times.
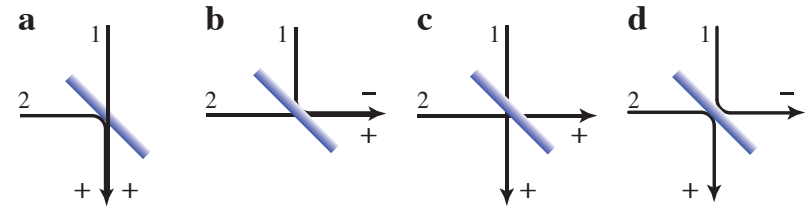

FIG. 8: Two impinging photons lead to four possible photon distributions at the beam-splitter output. In the first two cases (a) and (b) the photons would be found together. In the remaining two cases (c) and (d) the photons would leave the beamsplitter through different ports. Since the quantum states of the cases (c) and (d) show different signs, they interfere destructively.

As a remedy, in case of a photon duration that is very long compared to the detector time resolution, the detection time of a photon can also be measured within the single-photon envelope by recording the time delay between photodetections [56-58]. This time-resolved investigation of two-photon interference effects is a probate way to determine the coherence time.

We assume that two independent photon streams impinge on a symmetric beamsplitter such that two photons are superimposed, and we ask for the probabil- ity to detect the photons of each pair in different output ports of the beamsplitter. As we shall see, the joint detection probability is zero for identical photons. With polarisation-entangled photon pairs emitted from a down-conversion source, this effect has first been used to test the violation of Bell's inequality by joint photodetections [59], and one year later for measuring the delay between two photons with sub-picosecond precision [55]. Two-photon interference phenomena have also been employed to test the indistinguishability of independently generated single photons $[53,57]$.

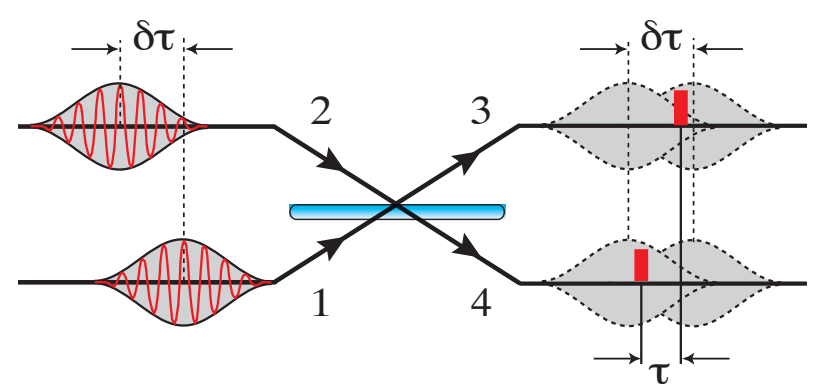

FIG. 9: Two single-photon wave packets impinge with a relative delay of $\delta \tau$ on ports ' 1 ' and ' 2 ' of a beamsplitter. The pulse duration is longer than the time resolution of the detectors monitoring ports ' 3 ' and ' 4 ' of the beamsplitter. Therefore, the time difference, $\tau$, between two photon detections must be taken into account in the analysis of the photon statistics.

As a starting point for the following discussion, we review the phenomenon in the number-state picture, i.e. we consider two photons that impinge on the entrance ports labelled ' 1 ' and ' 2 ' of the beam splitter shown in Fig. 9. This situation is described by the quantum state $\left|1_{1} 1_{2}\right\rangle=\hat{a}_{1}^{\dagger} \hat{a}_{2}^{\dagger}|00\rangle$. The effect of the beamsplitter on the field is usually described by the unitary transformations,

$$
\begin{array}{ll}
\hat{a}_{1}^{\dagger}=\frac{1}{\sqrt{2}}\left(\hat{a}_{3}^{\dagger}+\hat{a}_{4}^{\dagger}\right), & \hat{a}_{2}^{\dagger}=\frac{1}{\sqrt{2}}\left(\hat{a}_{3}^{\dagger}-\hat{a}_{4}^{\dagger}\right), \\
\hat{a}_{3}^{\dagger}=\frac{1}{\sqrt{2}}\left(\hat{a}_{1}^{\dagger}+\hat{a}_{2}^{\dagger}\right), & \hat{a}_{4}^{\dagger}=\frac{1}{\sqrt{2}}\left(\hat{a}_{1}^{\dagger}-\hat{a}_{2}^{\dagger}\right) .
\end{array}
$$

If we use these relations to express the initial state, we immediately see that this leads to an entangled state with either both photons in one or the other output,

$$
\begin{aligned}
\left|1_{1} 1_{2}\right\rangle=\hat{a}_{1}^{\dagger} \hat{a}_{2}^{\dagger}|00\rangle & \rightarrow \frac{1}{2}\left(\hat{a}_{3}^{\dagger}+\hat{a}_{4}^{\dagger}\right)\left(\hat{a}_{3}^{\dagger}-\hat{a}_{4}^{\dagger}\right)|00\rangle \\
& \rightarrow \frac{1}{\sqrt{2}}\left(\left|2_{3} 0_{4}\right\rangle-\left|0_{3} 2_{4}\right\rangle\right) .
\end{aligned}
$$

In simple physical terms, this can be also understood as destructive interference of the two possible paths leading to photons in different output ports, as illustrated by Fig. 8.

\section{Step-by-step analysis}

For a quantitative analysis, we now consider freerunning photons in the space-time domain. No boundary conditions restrict the photons to specific frequency 
modes, and we are free to define operators $\hat{a}_{k}^{\dagger}$ and $\hat{a}_{k}$ that create or annihilate photons in arbitrarily chosen spatio-temporal modes (labelled by the index $k$ ). In general, these modes can be defined by a one-dimensional spatio-temporal mode function

$$
\zeta_{k}(t)=\epsilon_{k}(t) e^{-i \phi_{k}(t)} .
$$

Beside an arbitrary phase evolution $\phi_{k}(t)$, the mode function incorporates an amplitude envelope $\epsilon_{k}(t)$, which we assume to be normalised so that $\int d t\left|\epsilon_{k}(t)\right|^{2}=1$. To analyse the effect of the beamsplitter, we consider the occupied input modes described by $\zeta_{1}(t)$ and $\zeta_{2}(t)$, and we then consider one photon detection after the other. That is we ask for the probability to detect a photon in output port ' 4 ' at time $t_{0}+\tau$ conditioned on a photon detection in output port ' 3 ' at time $t_{0}$. The probability to find a photon at time $t_{0}$ in port ' 3 ' simply reads

$$
\begin{aligned}
P_{3}\left(t_{0}\right) & =\left.\left\langle\Psi_{i n}\left|\hat{a}_{3}^{\dagger} \hat{a}_{3}\right| \Psi_{i n}\right\rangle\right|_{t_{0}} \\
& =\frac{1}{2}\left(\left|\epsilon_{1}\left(t_{0}\right)\right|^{2}+\left|\epsilon_{2}\left(t_{0}\right)\right|^{2}\right),
\end{aligned}
$$

where $\left|\Psi_{i n}\right\rangle=\left|1_{1} 1_{2}\right\rangle$ is the initial state. This expression includes no interference, since the initial product state is composed of single-photon states that have no relative phase. However, observing a photon at $t_{0}$ reduces the remaining quantum state to $\left|\Psi_{\text {cond }}\right\rangle$, which can also be seen as a state conditioned on the detection of a photon in port ' 3 ' at time $t_{0}$. It is obtained by applying the annihilation operator $\hat{a}_{3}$ to $\left|\Psi_{i n}\right\rangle$, which yields

$$
\left|\Psi_{\text {cond }}\right\rangle \propto \zeta_{2}\left(t_{0}\right)\left|1_{1} 0_{2}\right\rangle+\zeta_{1}\left(t_{0}\right)\left|0_{1} 1_{2}\right\rangle .
$$

This corresponds to a single photon impinging on the beamsplitter along two paths, with amplitude and phase relations given by $\zeta_{2}\left(t_{0}\right)$ and $\zeta_{1}\left(t_{0}\right)$. The result therefore is classical interference, with both paths in phase at $t_{0}$, and subsequent phase evolution according to $\zeta_{1}(t)$ and $\zeta_{2}(t)$. The probabilities to detect the second photon in output ports ' 3 ' or ' 4 ' at time $t_{0}+\tau$ therefore oscillates, so that the joint photon-detection probability for a first detection in output port ' 3 ' at time $t_{0}$ and a subsequent photon detection in output port ' 4 ' at time $t_{0}+\tau$ reads

$$
P_{34}\left(t_{0}, \tau\right)=\frac{1}{4}\left|\zeta_{1}\left(t_{0}+\tau\right) \zeta_{2}\left(t_{0}\right)-\zeta_{2}\left(t_{0}+\tau\right) \zeta_{1}\left(t_{0}\right)\right|^{2} .
$$

It is quite remarkable that the step-by-step analysis of two-photon interference reveals its equivalence to classical interference, with the only difference being the conditioning on the detection of a first photon. Note also that the sequence of photo detections is irrelevant, and for negative detection-time delay, $\tau$, which corresponds to a reverse order of photon detections, the same result is obtained.

\section{Identical and distinguishable photons}

From Eq. (27), we can see that the joint detection probability is always zero for identical photons, i.e. for

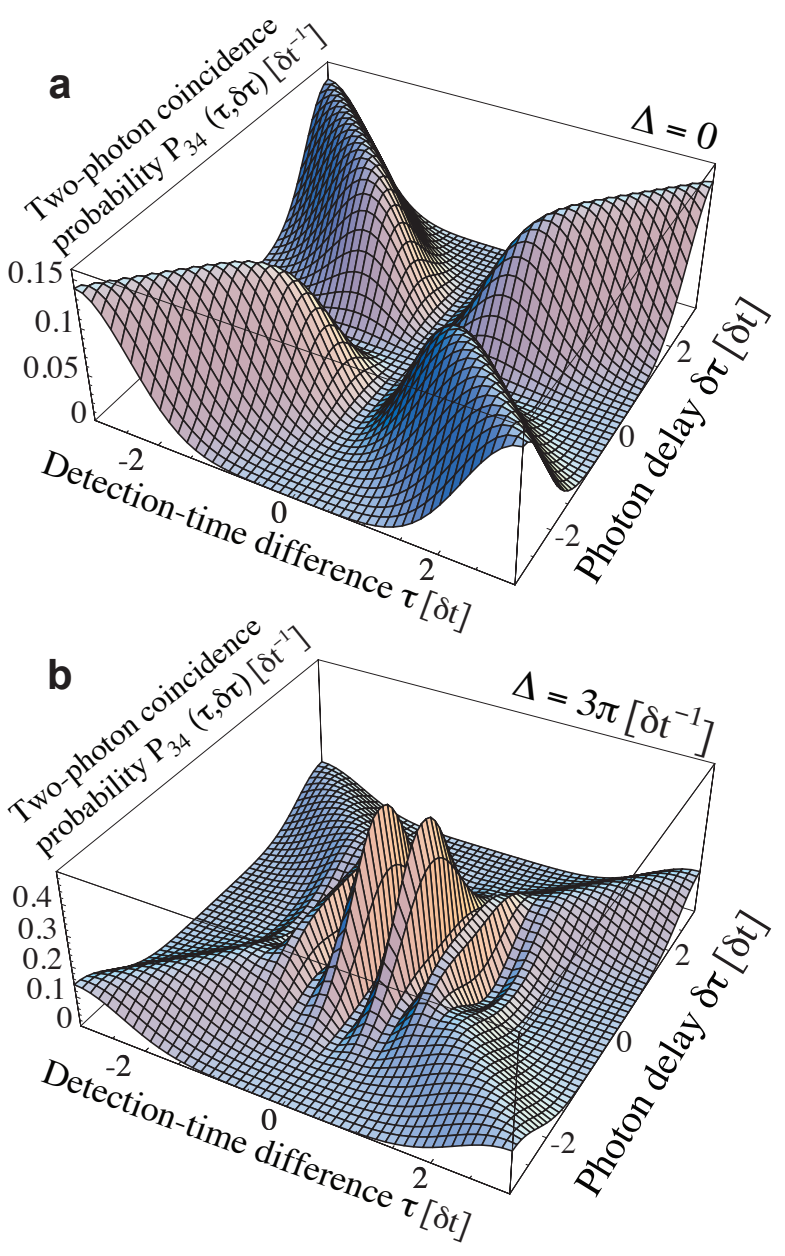

FIG. 10: Joint probability, $P_{34}$, for the detection of photon pairs as a function of the relative delay between the two impinging photon wave packets, $\delta \tau$, and the difference between photon-detection times, $\tau$ for the frequency differences $\Delta=0$ and $\Delta=3 \pi \delta t^{-1}$. All times differences are given in units of the photon-pulse duration $\delta t$.

$\zeta_{1}(t) \equiv \zeta_{2}(t)$, irrespective of the specific shape of the mode function. The situation is not that simple if the photons differ from one another. Still no joint detections occur simultaneously, i.e. $P_{34}(\tau=0) \equiv 0$, but the photons can be detected in different ports of the beam splitter as soon as $\tau \neq 0$.

To understand how slight differences between photons affect the joint detection probability, we consider Gaussian pulses of unit-time duration $\delta t$ (half 1 /e width) that impinge on the beamsplitter with a relative delay time $\delta \tau$ and a slight carrier-frequency difference $\Delta=\omega_{2}-\omega_{1}$. For these, evaluation of Eq. (27) yields a joint photondetection probability of

$$
\begin{gathered}
P_{34}(\tau, \delta \tau, \Delta)=\frac{1}{2 \sqrt{\pi}}(\cosh (2 \tau \delta \tau)-\cos (\tau \Delta)) \\
\times e^{-\delta \tau^{2}-\tau^{2}}
\end{gathered}
$$

which is averaged over $t_{0}$. This joint detection proba- 


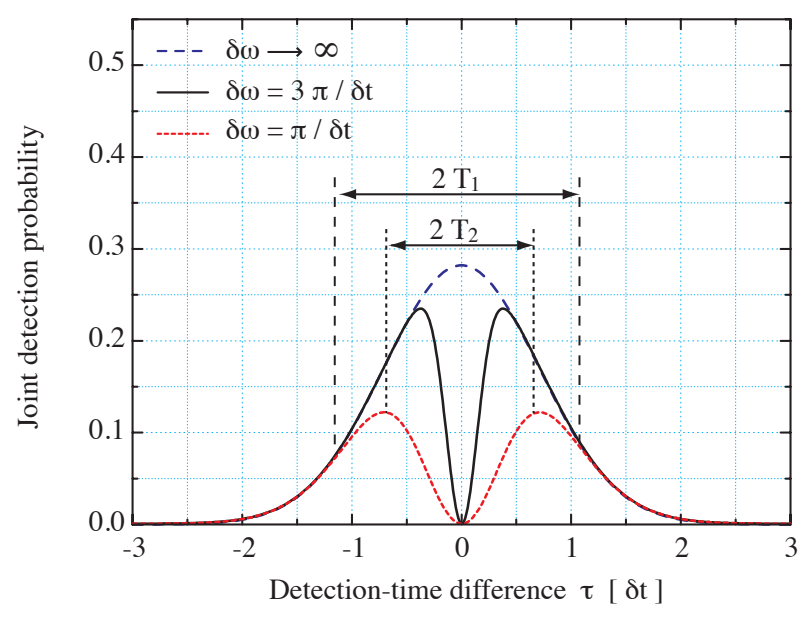

FIG. 11: Average joint detection probability, $\bar{P}_{34}$, as a function of the detection-time difference, $\tau$, for simultaneously impinging photons, $\delta \tau=0$, of identical polarisation. The photons are subject to a frequency jitter of width $\delta \omega$.

bility is displayed in Fig. 10 as a function of the photondelay, $\delta \tau$, and the detection-time delay, $\tau$, for two different values of the frequency difference, $\Delta$. The case $\Delta=0$ is depicted in Fig. 10a. As expected, no coincidences are found if the photons arrive simultaneously, i.e. for $\delta \tau=0$, which is well explained by the model of two interfering indistinguishable photons. Moreover, even if the two photons are delayed with respect to each other, we see that simultaneous photon detections (with $\tau=0$ ) never occur. Figure 10b shows a situation where the two impinging photon wave packets have a frequency difference of $\Delta=3 \pi$, in units of $\delta t^{-1}$. Simultaneous detections (with $\tau=0$ ) still do not occur, although the two photons are now distinguishable. However, for simultaneously arriving photon wave packets (with $\delta \tau=0$ ), the coincidence probability now oscillates as a function of $\tau$ with the frequency difference $\Delta$. This beat note always starts at $\tau=0$, due to the in-phase starting condition imposed by the detection of the first photon.

\section{Frequency jitter}

With the in-phase starting condition not depending on the mutual frequency difference $\Delta$ between the photons, the coincidence probability at $\tau=0$ remains zero even in case of an inhomogeneous broadening of frequencies. To illustrate this, we assume a Gaussian frequency distribution of bandwidth $\delta \omega$ (half width at $1 / e$ maximum), which yields an average photon detection probability of

$$
\bar{P}_{34}(\tau, \delta \tau=0, \delta \omega)=\frac{e^{-\tau^{2}}}{2 \sqrt{\pi}}\left(1-e^{-\tau^{2} \delta \omega^{2} / 4}\right) .
$$

As shown in Fig. 11, a $2 / \delta \omega$-wide dip is found in the two-photon detection probability. The dip goes down to zero for $\tau=0$, and the bandwidth of the photons is only affecting its width. Hence the dip-width seems a good measure for the mutual coherence time (or reciprocal bandwidth) of the photons.

It should be noted here that the above is only applicable for photons long compared to the detector timeresolution. If solid state systems are considered, the light pulses are usually far too short to measure any detectiontime difference. In this latter case, the coincidence probability is only a function of the relative photon delay, $\delta \tau$, and is given by

$$
\left\langle P_{34}(\delta \tau)\right\rangle_{\tau}=\frac{1}{2}\left(1-\frac{2 \cos ^{2} \varphi}{\sqrt{4+\delta t^{2} \delta \omega^{2}}} e^{-\delta \tau^{2} / \delta t^{2}}\right) .
$$

Any frequency jitter, $\delta \omega$, now leads to a decreased depth of the Gaussian-shaped dip, while the width of this dip is not affected and always identical to the photon duration.

\section{Time jitter}

Even perfectly identical photons could be subject to a jitter in their mutual arrival time at the beam splitter. This gives rise to a relative delay between the two mode functions, and hence to a non-zero joint detection probability for $\tau>0$. Assuming a Gaussian arrival-time distribution of width $\Delta \tau$, the average $P_{34}$ shows again a central dip reaching zero, which now has the width

$$
\sqrt{\delta t^{2}+\delta t^{4} / \Delta \tau^{2}}
$$

As before, time-resolved two-photon interference can be used to determine the variation in the emission time of the photons. However, since the shapes of the joint detection probabilities for frequency and emission-time jitter are identical, it is in general not possible to distinguish between the two broadening mechanisms. Nonetheless, one can determine the maximum values for both, as well as all pairs of frequency and emission-time jitter combinations matching the data. This is discussed in [58].

In this section, we have shown that the HanburyBrown \& Twiss and Hong-Ou-Mandel techniques constitute two excellent tools to characterise single photons. In the latter technique, two photons are superimposed on a beamsplitter and the joint detection probability is in the two output ports of the beam splitter measured as a function of the arrival plus detection-time difference of the photons. For identical photons, the joint detection probability is expected to be zero. Variations of the spatiotemporal modes of the photons lead to joint photodetections except for zero detection-time difference. Therefore the joint detection probability shows a pronounced dip. From the width of this dip, one can estimate the maximum emission-time jitter and the minimum coherence time of the photons. Figure 12 illustrates how to use the Hong-Ou-Mandel technique to characterise a single photon emitter. The first of a pair of successively emitted photons is send along an optical delay line to 


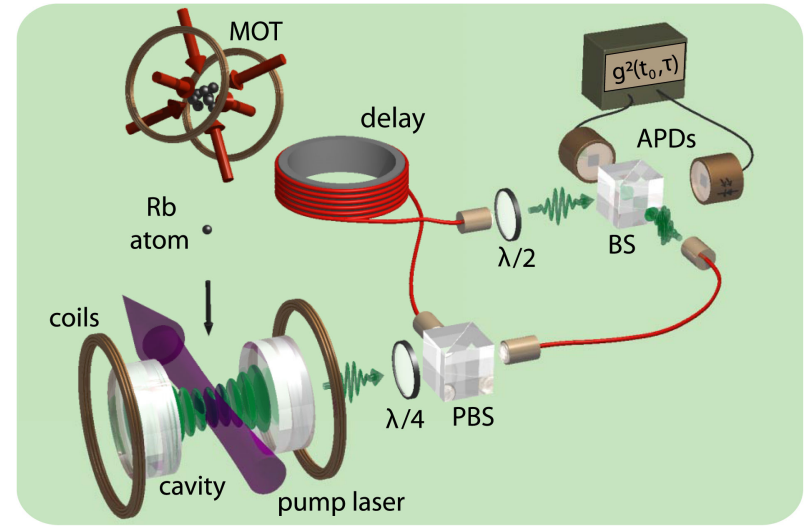

FIG. 12: Typical setup for performing a two-photon interference experiment to characterise a single photon emitter $[57,60]$. The first of a pair of successively emitted photons is sent along an optical delay line, e.g. a fibre of several $100 \mathrm{~m}$ length, to arrive simultaneously with the second photon at a beam splitter. Coincidences in the output ports of this beamsplitter are recorded. Rotating the relative polarisation of the interfering photons is a way to obtain a reference signal, as light of perpendicular polarisation yields no interference.

arrive simultaneously with the second photon at a beam splitter. Hence the joint detection probability is primarily affected by the mutual difference between successive photons. Long-term drifts of spectral properties will have a negligible effect on the signal.

\section{ROUTES TO SINGLE-PHOTON SOURCES}

In this section we review different types of singlephoton sources that have been implemented to date where the Purcell effect plays a major role in enhancing the emission from single-atoms, single-ions, or artificial atoms (quantum dots) placed in optical cavities. In order to compare each of the implementations in terms of their ability to deliver well-prepared single-photons, and their ease of operability, we introduce a classification scheme based on the three classes of cavity-QED emission schemes discussed in section 3. Along with a discussion of the pros and cons of the different sources we also provide a table of benchmarks for some selected realisations. We furthermore give a short view on the many types of cavities which advance in technology has made available in recent years.

Hitherto, undoubtedly the most popular technique to generate single photons is parametric down-conversion in non-linear crystals, which can mainly be explained by its simple operation and low cost. Even though it can quite easily produce high-rate streams of photon-pairs, and thus also single-photons by heralding one photon on the detection of the other - resulting in low values of the $g^{(2)}$ autocorrelation, it suffers from the major drawback of not producing photons deterministically, but more or less at random times due to the spontaneous nature of the generation process. To some extent this can be resisted by post-selection, but it is still not a very efficient method. In addition, the frequency bandwidth originating from the phase-matching conditions of parametric down-conversion is in general very broad on the scale of atomic transitions, and thus unsuitable for many applications like, e.g., atom-based qubit memories.

During the last decade also other photon generation schemes have been demonstrated, such as a single-photon turnstile device based on the Coulomb blockade mechanism in a quantum dot [65], the fluorescence of a single molecule $[66,67]$, or a single colour centre (Nitrogen vacancy) in diamond $[45,68]$, or the photon emission of a single quantum dot into free space [44, 69, 70]. All these new schemes emit photons upon an external trigger event. However, the photons are spontaneously emitted into many modes of the radiation field, i.e. into all directions, and they usually show a very broad energy distribution. For the same reason, the emission process cannot be described by a Hamiltonian evolution. Hence the process is hardly reversible, and does not allow for coherent quantum state mapping from source to photon and back. This does not hamper one from using these photons for quantum cryptography and communication, but it represents a major obstacle to all applications in quantum computing or quantum networking. As discussed in the previous sections, most of these limitations can be overcome by cavity-enhanced emission techniques into welldefined modes of the radiation field. The remainder of this section will focus on these.

In section III, we have outlined three different ways of producing photons from a cavity-based system. Fig. 13 illustrates these three schemes, along with the basic features of the underlying physical methods and the properties of the photons obtained. All cavity-based photon sources realised so far nicely fit into one of these three categories, which one therefore can use to classify a large variety of existing single-photon sources. Apart from the applied scheme, further relevant parameters are the cavity's mode volume $V$, its finesse $\mathcal{F}$, coupling and damping rates $\left\{g_{0}, \kappa, \gamma_{\perp}\right\}$, cooperativity $C=g_{0}^{2} /\left(2 \kappa \gamma_{\perp}\right)$, and all the benchmark parameters characterising the photons produced, namely their coherence time $\tau_{c}$, the triggerpulse rate $R_{T}$ and the efficiency $\eta$, which is the probability for a photon emission upon a single trigger pulse sent to the system. With some of the sources showing a blinking or intermittent behaviour of operation, both $R_{T}$ and $\eta$ must refer to the active mode of operation to allow for any comparison of different implementations. For the same reason, it is rather inconvenient to simply ask for $g^{(2)}(0)$. Instead, the anti-bunching ratio $g^{(2)}(0) / g^{(2)}(1)$, defined as a ratio of pulse areas in the autocorrelation function, i.e. the area of the pulse around $\tau=0$ to the area of its nearest neighbours, is more appropriate [50]. Table I is listing all these properties and parameters for several different implementations of single-photon sources, along with the type of cavity being used. We will 


\begin{tabular}{|c|c|c|c|c|c|c|c|c|c|c|c|c|}
\hline Type & Class & Cavity & $\begin{array}{c}V \\
{\left[\mu \mathrm{m}^{3}\right]}\end{array}$ & $\begin{array}{c}\mathcal{F} \\
\text { or } Q^{\dagger}\end{array}$ & $\begin{array}{l}g_{0} / 2 \pi \\
{[\mathrm{MHz}]}\end{array}$ & $\begin{array}{l}\gamma_{\perp} / 2 \pi \\
{[\mathrm{MHz}]}\end{array}$ & $\begin{array}{c}\kappa / 2 \pi \\
{[\mathrm{MHz}]}\end{array}$ & $\mathcal{C}$ & $\frac{g^{(2)}(0)}{g^{(2)}(1)}$ & $\begin{array}{c}\tau_{c} \\
{[\mathrm{~ns}]}\end{array}$ & $\begin{array}{c}R_{T} \\
{[\mathrm{MHz}]}\end{array}$ & $\eta$ \\
\hline $\operatorname{atom}^{[52]}$ & III & FPI & $10^{4}$ & $4 \times 10^{5}$ & 16 & 2.6 & 4.2 & 10 & 0.05 & $10^{2}$ & 0.1 & 0.6 \\
\hline atom $[60]$ & III & FPI & $10^{6}$ & $6 \times 10^{4}$ & 3.0 & 3.0 & 1.3 & 1.2 & 0.07 & 470 & 0.5 & 0.4 \\
\hline $\operatorname{qdot}^{[61]}$ & I & MD & $\sim 16$ & - & - & 80 & - & - & 0.29 & $\overline{3.4}$ & 82 & - \\
\hline $\operatorname{qdot}^{[53]}$ & I & MP & $5-80$ & $1000^{\dagger}$ & $10^{4}$ & 80 & $3 \times 10^{5}$ & 2.5 & $<0.04$ & $\underline{0.2}$ & 77 & 0.0016 \\
\hline $\operatorname{qdot}^{[62,63]}$ & I & $\mathrm{PC}$ & 1 & $3000^{\dagger}$ & 5000 & 80 & $10^{5}$ & 2.5 & 0.54 & $\overline{0.06}$ & 80 & - \\
\hline ion $[64]$ & II/III & FPI & $10^{6}$ & $7 \times 10^{5}$ & 1.6 & 11 & 0.05 & 2.3 & $<0.02$ & $<10^{5}$ & 0.002 & 0.88 \\
\hline $\operatorname{ion}^{[34]}$ & II/III & FPI & $10^{6}$ & $10^{4}$ & 1.4 & 0.8 & 1.2 & 1.0 & $<0.02$ & $10^{2}$ & 0.1 & 0.08 \\
\hline
\end{tabular}

TABLE I: Typical performances for different types and classes of cavity-based systems: single atoms, single ions, and solidstate (quantum dots). Cavity parameters; $V$ : mode volume; $\mathcal{F}$ : finesse (or $Q$-factor); $g_{0}$ : atom-cavity coupling; $\gamma_{\perp}:$ polarisation decay rate; $\kappa$ : cavity-field decay rate; $\mathcal{C}$ : cooperativity (Purcell factor $f=2 C$ ). Photon benchmark parameters (only if the cavity has been used for single-photon generation); $g^{(2)}(0) / g^{(2)}(1)$ : ratio of pulse areas in the intensity auto-correlation (see text); $\tau_{c}$ : coherence time (underlined values verified by two-photon interference); $R_{T}$ : single-photon trigger rate; $\eta$ : combined efficiency for single-photon generation and escape; ( $R_{T}$ and $\eta$ given for the operational mode in case of blinking sources). The cavity types stated in the table are FPI: Fabry-Perot; MD: micro disk; MP: micro pillars; PC: photonic crystal. Note that some of the listed parameters were not given in the original publications and therefore are inferred from other information, like, e.g., $g_{0}$ for the three quantum-dot systems. Also the efficiency for the actual photon generation and escape from the cavity was not specified in all cases.

now discuss these approaches in more detail.

\section{A. Neutral single atoms}

A straightforward implementation of a cavity-based single-photon source consists of a single atom placed between two separate cavity mirrors, with a stream of laser pulses travelling perpendicular to the cavity axis to trigger photon emissions. The most simplistic approach to achieve this is by sending a dilute atomic beam through the cavity, with an average number of atoms in the mode far below one. However, for a thermal beam, the obvious drawback would be an interaction time between atom and cavity far too short to achieve any control on the exact photon emission time. Hence cold (and therefore slow) atoms are required to overcome this limitation. One of us actually followed this route [33], using a magneto-optical trap to cool a cloud of atoms to a few tens of $\mu \mathrm{K}$ at a distance of $20 \mathrm{~cm}$ above the cavity. Atoms released from the trap eventually fall through the cavity, and interact with its mode for $17.5 \mu \mathrm{s}$. Within this limited interaction time, up to seven single-photon emissions have been triggered. Fig. 14 illustrates this setup, together with some possibilities of using either transitions between hyperfine or Zeeman substates in rubidium to establish the adiabatic passage technique discussed in section III C to generate single photons.

Bursts of single photons are emitted from the cavity whenever a single atom passes its mode, and strong antibunching is found in the photon statistics, as shown in Fig. $7 \mathrm{c}+$ d. Nonetheless, with the atoms arriving at random times, no sub-Poissonian behaviour is found. A possible way to overcome this minor drawback is by conditioning the experiment on the actual presence of an atom in the cavity [49]. A good example for doing so is the characterisation of the photons by two-photon interference discussed in section IV B. For these experiments, pairs of photons are needed that meet simultaneously at a beamsplitter. As discussed previously, see Fig. 12, this is achieved by delaying the first photon of a pair of successively emitted photons. With the occurrence of these photon pairs being the precondition to observe any correlation and the probability for successive photon emissions being vanishingly small without atoms, the presence of an atom is actually assured whenever data is recorded.

For instance, two-photon interference has been studied in a time-resolved manner in these experiments. For photons of $1 \mu$ s duration, a dip-width (and therefore coherence time) of $470 \mathrm{~ns}$ is found, which is about one order of magnitude longer than the decay rate of either cavity or atom. This proves that the photon's coherence is to a large extend controlled by the Raman process, without being limited by the decay channels within the system. Figure 15 shows the coincidences measured behind the beam splitter of the Hong-Ou Mandel experiment as a function of the detection time delay. In this particular case, ${ }^{87} \mathrm{Rb}$-atoms are excited in a way that the cavity emits photons of alternating polarisation [60]. Coincidences are of course found if photons of perpendicular polarisation impinge on the beam splitter, as they do not interfere. This signal constitutes a nice reference that can easily be compared to the coincidence rate found if the photons are nearly indistinguishable, i.e. with parallel 


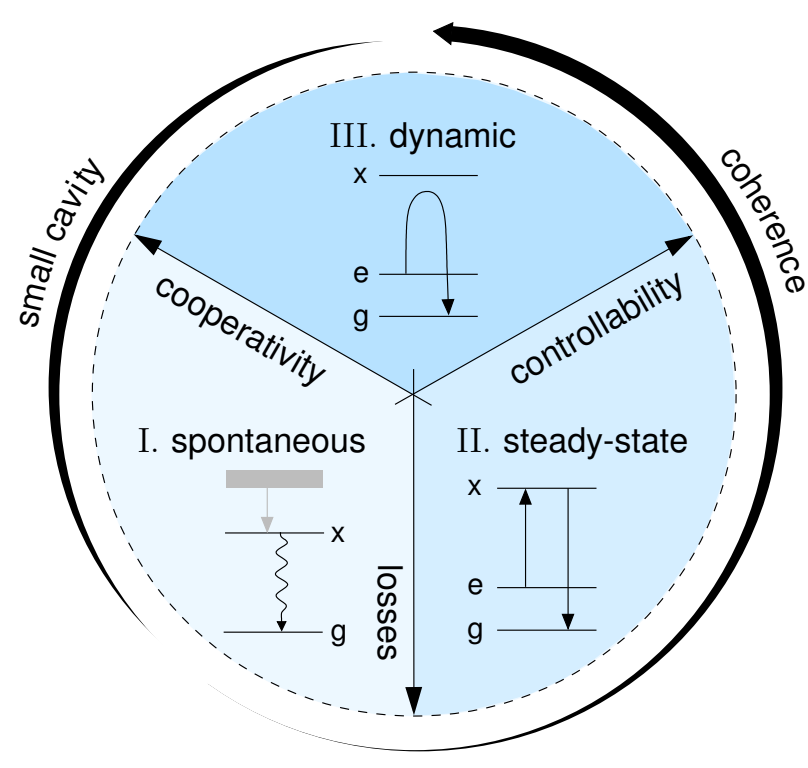

FIG. 13: A simple classification scheme for cavity-based single-photon sources. Quantum dots operate today mainly as class I, with very small cavity volumes and hence very high coupling factors, but suffer losses from incoherent atomic and cavity decay. With ions, a substantially higher degree of coherence can be reached in class II to III. In class II, the idea is to get the photon out as fast as possible since one cannot avoid spontaneous emission losses. With the even smaller cavity volumes available to atoms, full dynamic control is reached in class III with high cooperativity parameters. Since spontaneous losses are then avoided, we can pull the photon out slower with greater probability. Ideally, future advances allow to combine single atoms with photonic crystal or fibre-tip cavities, such that class III operation at very small cavity volumes become possible.

polarisation. As can be seen from Fig. 15, almost no more coincidence counts are found in this case, which proves the mutual coherence of successively emitted photons.

Only lately, refined versions of this type of photon emitter have been realised, with a single atom held in the cavity using a dipole-force trap. McKeever et al. [52] managed to hold a single Cs atom in the cavity with a dipole-trapping beam running along the cavity axis, while Hijlkema et al. [51] are using a combination of dipole trapping beams running perpendicular and along the cavity to catch and hold a single $\mathrm{Rb}$ atom in the cavity mode. As illustrated in Fig. 16, the trapped atom is in both cases exposed to a sequence of laser pulses alternating between triggering the photon emission, cooling and repumping the atom to its initial state to repeat the sequence. As the atom is trapped, the photon statistics is no more affected by fluctuations in the atom number and therefore is sub-Poissonian (see Fig. 7e). Moreover, with trapping times for single atoms up to a minute, a quasi-continuous bit-stream of photons is obtained.

The major advantage of using neutral atoms as pho-

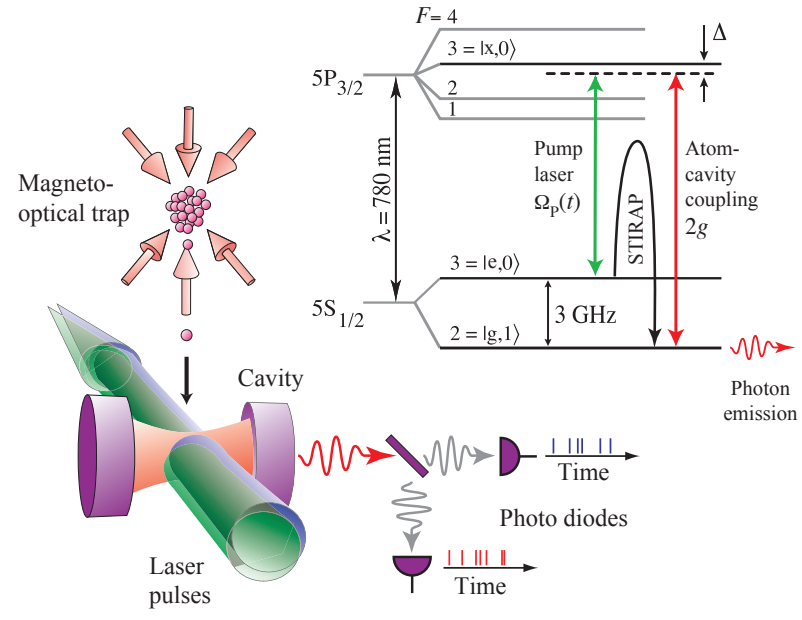

FIG. 14: Scheme of the first single-photon source based on an atom-cavity system [33]. A cloud of atoms released from a magneto-optical trap falls through a cavity $20 \mathrm{~cm}$ below. Laser pulses controlling the emission travel perpendicular to the cavity axis. The light is analysed using a Hanbury-Brown \& Twiss setup with a pair of photodiodes of $50 \%$ quantum efficiency. The relevant energy levels and transitions in ${ }^{85} \mathrm{Rb}$ are shown as well. The atomic states labeled $|e\rangle,|x\rangle$ and $|g\rangle$ are involved in the Raman process, and the states $|0\rangle$ and $|1\rangle$ denote the photon number in the cavity.

ton emitters in Fabry-Perot type cavities is that a relatively short cavity (some $100 \mu \mathrm{m}$ ) of high finesse (between $10^{5}$ and $10^{6}$ ) can be used. As stated in table I, one thus obtains strong atom-cavity coupling, and the photon generation can be driven either in the steady-state regime (class II) or dynamically by vacuum-stimulated Raman adiabatic passage (V-STIRAP, class III). This allows one to control the coherence properties and the shape of the photons to a large extent, as discussed in section 3.4. Photon generation efficiencies as high as $40 \%$ have been demonstrated with these systems. Furthermore, based on the excellent coherence properties, first applications such as atom-photon entanglement and atom-photon state mapping $[9,10]$ have recently been demonstrated. At first glance, a significant problem in all these experiments seems the thermal motion of the atom in the surrounding dipole trap. The region explored by the atom brings it out of the Lamb-Dicke regime, and the atomic motion in the trap leads to variations of the atomic levels due to the dynamic Stark shift. However, these effect are fortunately not detrimental to transitions adiabatically driven between atomic ground states, as has been largely discussed in [71].

Apart from the above Fabry-Perot type cavities, many other micro-structured cavities have been explored during the last years. These usually provide a much smaller mode volume and hence boost the atom-cavity coupling strength by about an order of magnitude. However, this goes hand-in-hand with increased cavity losses and thus a much larger cavity linewidth, which might be in conflict 


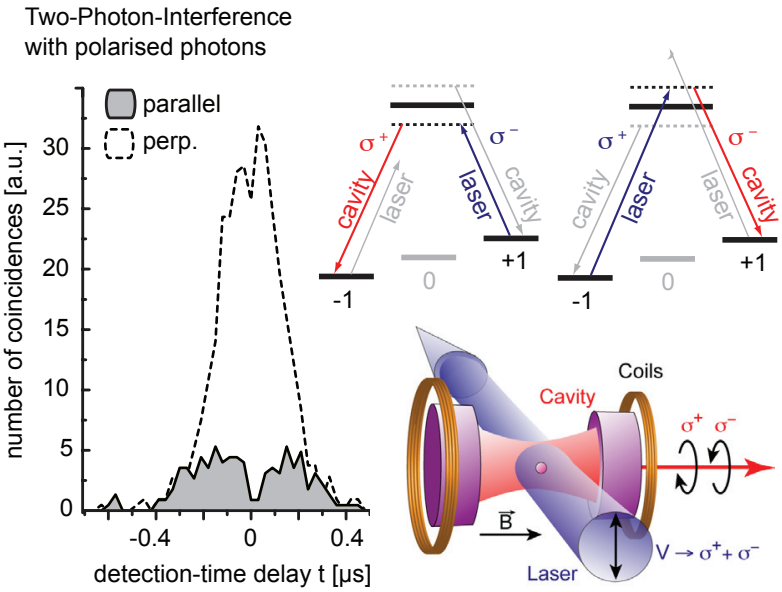

FIG. 15: Time-resolved two-photon interference signal for polarised photons emitted from an atom-cavity system [60], recorded with the setup depicted in Fig. 12. The dotted line shows the correlations found when distinguishable photons of perpendicular polarisation meet on the beam splitter, while the solid line depicts the correlations found if the photon polarisation is parallel. The inset shows the level scheme in ${ }^{87} \mathrm{Rb}$ used to generate photons of alternating polarisation by driving Raman transitions in between the magnetic sublevels $m_{F}= \pm 1$ of the $F=1$ hyperfine state. As illustrated, a magnetic field along the cavity axis is used to lift the degeneracy between magnetic sublevels.

with the desired addressing of individual atomic transitions. Among the most relevant new developments are fibre-tip cavities, which use dielectric Bragg stacks at the tip of an optical fibre as cavity mirrors [72, 73]. Due to the small diameter of the fibre, either two fibre tips can be brought very close together, or a single fibre tip can be complemented by a micro-structured mirror on a chip to form a high-finesse optical cavity. A slightly different approach are ring-cavities realised in solid state, guiding the light in a whispering gallery mode. An atom can be easily coupled to the evanescent field of the cavity mode, provided it can be brought close to the surface of the substrate. Nice examples are microtoroidal cavities realised at the California Institute of Technology [74, 75], and bottle-neck cavities in optical fibres [76]. These cavities have no well-defined mirrors and therefore no output coupler, so one usually arranges for emission into well-defined spatio-temporal modes via evanescent-field coupling to the core of an optical fibre.

We would like to remind the reader at this point that a large variety of other exciting cavity-QED experiments has been performed that were not aiming at a singlephoton emission in the optical regime. Most important amongst these are the coupling of Rydberg atoms [77] or superconductive SQUIDs [78] to microwave cavities, which is also a well-established way of placing single photons into a cavity using either $\pi$ pulses [79] or dark resonances [80]. Also the coupling of ultracold quantum gases to optical cavities has been studied extensively $[72,81]$,
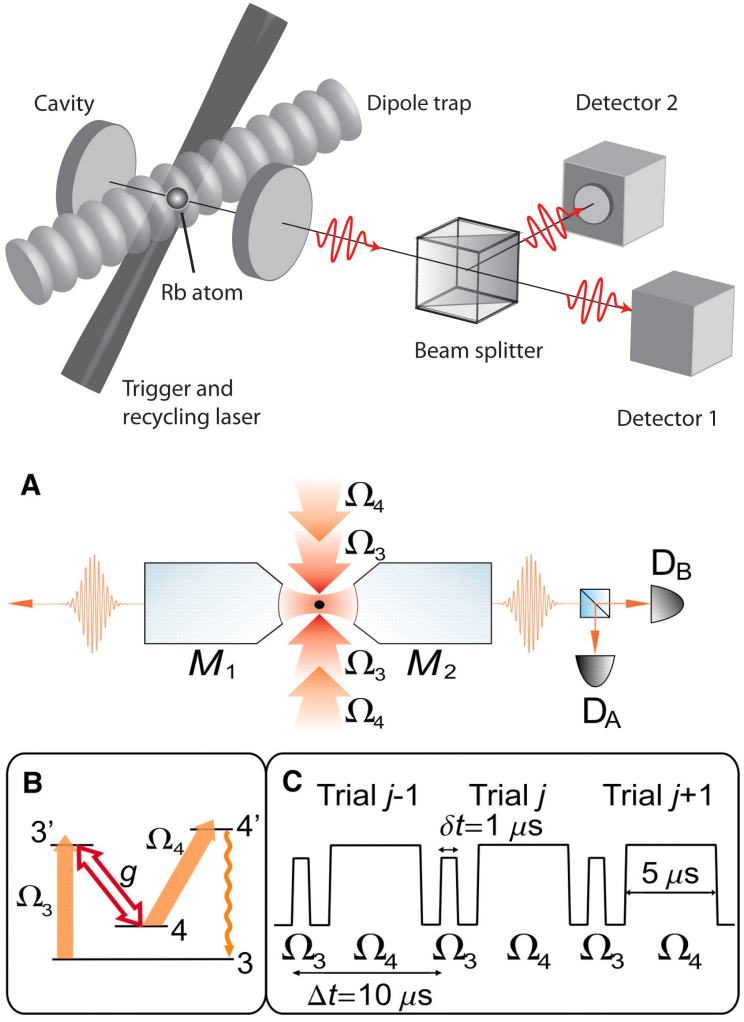

FIG. 16: Atom-cavity systems with a single atom at rest in the cavity mode. Top: One of us has been using a dipole trap running perpendicular to the cavity axis is holding a single rubidium atom trapped in the cavity [51]. The cavity is asymmetric, and photons emitted through its output coupler are directed to a pair of photon counters to record the second-order correlation function of the photon stream. Bottom: J. McKeever et al. [52] are using a dipole trap running along the cavity axis is used to hold a single caesium atom in the cavity. The cavity is symmetric, and only half the photons are directed towards a pair of detectors for analysing the photon statistics (From J. McKeever et al., Science 303, 1992-1994 (2004). Reprinted with permission from AAAS). In both cases, the trapped atom is exposed to a sequence of laser pulses that trigger the photon emission, cool the atom, and re-establish the initial condition by optical pumping.

and has proven to be a useful method to acquire information on the atom statistics. Last but not least, large efforts have been made to study cavity-mediated forces on either single atoms or atomic ensembles [82-87], which eventually lead to the development of cavity-mediated cooling techniques.

\section{B. Trapped single ions}

Although neutral-atom systems have their advantages for the generation of single photons, such experiments are sometimes hampered by fluctuating atomcavity coupling and multi-atom effects. Also trapping times are still lim- 
ited in the intra-cavity dipole-trapping of single atoms. A possible way around these problems is to use a strongly localised single ion in an optical cavity, as has first been demonstrated by M. Keller et al. [34]. In their experiment, an ion is optimally coupled to a well-defined field mode, resulting in the reproducible generation of singlephoton pulses with precisely defined timing. The stream of emitted photons is uninterrupted over the storage time of the ion, which, in principle, could last for several days.

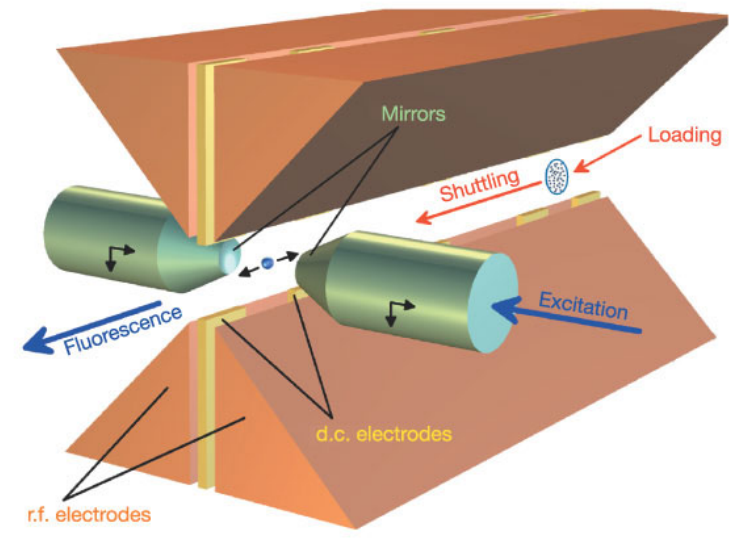

FIG. 17: Arrangement of ion-trap electrodes and cavity in the experiment by M. Keller et al. [34]. The ion is shuttled to the cavity region after loading. Upon excitation of the ion from the side of the cavity, a single photon gets emitted into the cavity mode. Reprinted by permission from Macmillan Publishers Ltd: Nature (G. Guthöhrlein et al. [88]), copyright 2001.

The major difficulty in combining an ion trap with a high-finesse optical cavity comes from the dielectric cavity mirrors, which influence the trapping potential if they get too close to the ion. This effect might be detrimental in case the mirrors get electrically charged during loading of the ion trap, e.g. by the electron beam used to ionise the atoms. Fig. 17 shows how this problem has been solved in [34] by shuttling the trapped ion from a spatially separate loading region into the cavity. Nonetheless, the cavity in these experiments is typically more than 10-20 $\mathrm{mm}$ long to avoid distortion of the trap. Thus the coupling to the cavity is weak, and albeit optimised pump pulses were used, the single-photon efficiency in [34] did not exceed $(8.0 \pm 1.3) \%$. This is in good accordance with theoretical calculations, which also show that the efficiency can be substantially increased in future experiments by reducing the cavity length. It is important to point out that the low efficiency does not interfere with the singleness of the photons. Hence the $g^{(2)}$ correlation function of the emitted photon stream corresponds to the one depicted in Fig. 7e, with $g^{(2)}(0) \rightarrow 0$. Only recently, H. G. Barros et al. [64] have demonstrated an improved version of an ion-cavity setup. Albeit their cavity is of similar length length, they were able to reach a single-photon efficiency of $(88 \pm 17) \%$, using a more favourable mode structure of the cavity and far-off resonant Raman transitions between magnetic sublevels of the trapped ion.

\section{Artificial atoms in solid-state}

Excellent reviews of photon sources based on artificial atoms in solid-state systems have been published by M. Oxborrow and A. G. Sinclair [89], B. Lounis and M. Orrit [90] and A. J. Shields [91]. Therefore we only discuss the basic features and key technologies here.

Quantum dots are often considered as artificial atoms par excellence, as they usually possess several discrete energy levels for electron-hole pairs, with optical transitions between these levels comparable to electronic transitions in atoms. In first experiments, self-assembled individual quantum dots have been exposed to either exciting laser pulses or to a continuous beam, and the fluorescence was collected and analysed using a microscope of high numerical aperture. While antibunching has been found in the light emitted from a single quantum dot [44, 69, 70], the isotropic emission of photons resulted in a very low photon-collection efficiency.

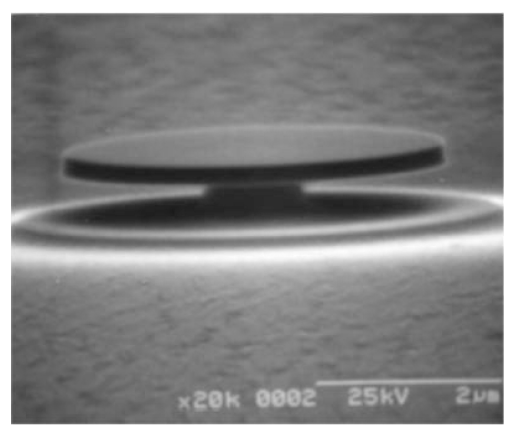

FIG. 18: The microdisk toroidal cavity used by P. Michler et al. [61] consists of a $5 \mu \mathrm{m}$-diameter disk and a $0.5 \mu \mathrm{m}$ post. The cavity has a whispering gallery mode running around the $200 \mathrm{~nm}$ thick GaAs disk, which is containing InAs quantum dots. From P. Michler et al., Science 290, 2282-2285 (2000). Reprinted with permission from AAAS.

To improve the probability for photon collection, several research teams successfully coupled individual quantum dots to micro-structured cavities, consisting either of micro disks guiding a whispering gallery mode [61] (see Fig. 18) or micro-pillars with Bragg-stacks of dielectric surfaces [53, 92-97]. Fig. 19 shows a scanning electron micrograph of such a pillar, along with a calculation of the electric field amplitude in the cavity mode. For all these systems, the cavity resonance frequency needs to be equal to a transition frequency of the quantum dot. Both can be altered by changing the temperature of the whole setup, but the tuning range is often insufficient. Nonetheless, the random scatter of resonance frequencies available within a large ensemble of micro cavities and quantum dots normally allows one to find a match- 
ing pair. Upon continuous or pulsed excitation, such a resonant pair of quantum-dot and cavity is emitting sub-Poissonian light, with a photon statistics similar to the one shown in Fig. 7(e). Furthermore, two-photon interference can be studied by delaying the first of two successively emitted photons, so that both meet on a beam splitter [53]. At zero time-delay between the impinging photons, the coincidence rate between the two beam-splitter exit ports shows a pronounced dip, which proves that independently generated photons are mutually coherent.

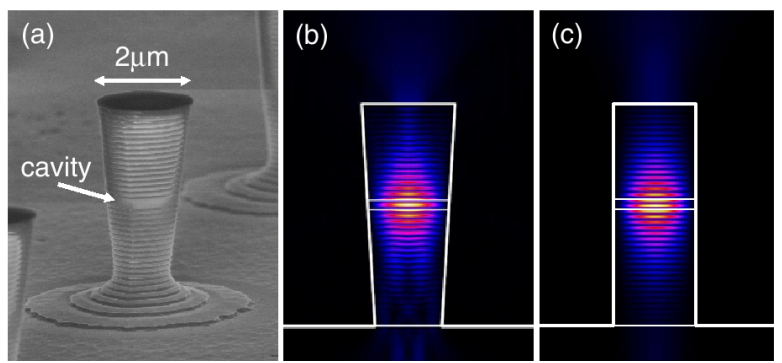

FIG. 19: (a) Micro-pillar cavities used by D. C. Unitt et al. [96] with a $2 \mu \mathrm{m}$ diameter. (b,c) show the calculated field amplitude of the cavity mode in the pillar. By courtesy of A. Shields (Toshiba UK), Copyright (2005) IOP Publishing Limited [96].

In a variant of the aforementioned micro-pillar experiments, M. Benyoucef et al. [98] were able to produce entangled photon pairs from a quantum dot coupled to a micro-pillar cavity. The two photons are emitted in a relaxation cascade and therefore generated almost simultaneously in a single step. We emphasise that this is substantially different from other approaches which produce entangled photons one-after-the-other [9, 10]. Very recently, more elaborate schemes for pulsed on-demand sources have been proposed [99], which might even lead to the emission of large photon clusters from single quantum dots. With the photon emissions being caused by a fast relaxation cascade, such a source would constitute a 'photon machine gun'.

Possible alternatives to micro-disk or micro pillar cavities are photonic-bandgap cavities, which consist of artificial defects in an otherwise regular photonic crystal. Such cavities have the potential to allow for an easy coupling of the light into optical fibres embedded on a chip surface, and have therefore been studied by a large number of research teams $[62,63,100,101]$. The defects in the photonic crystal give rise to local trapping of light, and thus form an effective cavity on the nano-scale. The mode volume of these cavities is typically of the order of $\lambda^{3}$, resulting in atom-cavity coupling strengths up to $100 \mathrm{GHz}$. To bring a quantum dot and a photonic-crystal cavity into resonance, the team around A. Imamoglu has developed a digital etching technique [62], which works by changing the radii of the crystal-forming holes, layer for layer of atoms, until the resonance condition is met. Fig. 20 shows the defect in the photonic crystal structure acting as high-Q cavity, and the modification of the spectrum in response to the cavity-etching process. As in experiments with micro-pillar cavities, photon-antibunching has been observed in the light scattered off the cavity.

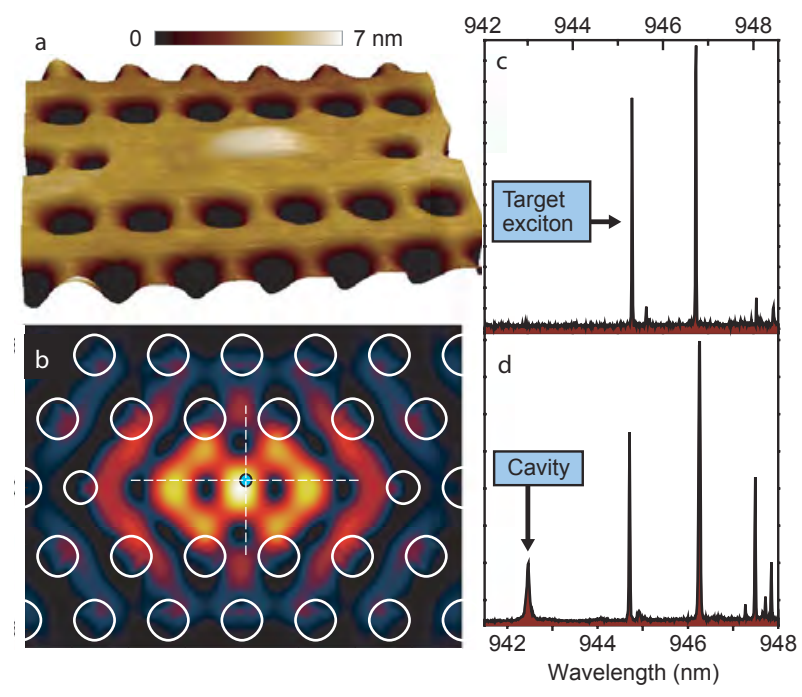

FIG. 20: Photonic crystal cavity coupled to a single buried quantum dot used by K. Hennessy et al. [63]. (a) AFM topography of the photonic crystal cavity aligned to a hill of material arising from a quantum dot. (b) Field intensity of the cavity mode, showing that the quantum dot overlaps the field maximum. (c+d) Photoluminescence spectra of a single quantum dot before and after cavity fabrication, showing emission from the cavity at $942.5 \mathrm{~nm}$. Reprinted by permission from Macmillan Publishers Ltd: Nature (K. Hennessy et al. [63]), copyright 2007 .

For many solid-state systems, the reduced lightcollection efficiency seems to be a substantial limitation. Some cavity designs have either no dedicated output coupler, or are plagued by a high jump in refractive index between bulk and outside world. Due to that, the efficiencies listed for quantum dots in table I are either very small or not stated at all. However, this does not constitute a principal limit, as promising proposals have been made for accessing the photons using integrated optics [102]. One might also think that using artificial atoms embedded in solid state cavities will outperform most other approaches, as the presence of a single emitter in the cavity mode is assured once and forever - which undoubtedly is the major advantage of the solid-state approach. However, no two quantum dots are equal, and mutual coherence between photons from different sources is hard to achieve. Moreover, the finesse of the solid-state cavities is roughly one order of magnitude smaller than the typical finesse obtained with dielectric mirrors, and the solid-state cavities also have a very tiny volume. This leads to a very fast photon loss from the cavity, combined with a very strong atom-cavity coupling. In turn, the timescale for the process is rather short, and no adiabatic control of the emission process is possible. The 
process typically starts with a driving laser pulse exciting the quantum dot to an energetically higher level, followed by a relaxation cascade down to the upper level used for photon emission. The last step in this cascade then is the cavity-enhanced emission of the desired photon. The emission into the cavity is therefore dominantly spontaneous (class I source), and the driving laser pulses have barely any influence on the shape or coherence properties of the photons. To some extend, this reduces the usefulness and in particular the scalability of present solid-state photon emitters.

Last but not least, we would like to emphasise that all the single-photon emitters we discussed so far require an optical excitation of the emitter, be it an atom, ion, or quantum dot. Very recently, A. J. Shields and D. A. Ritchie have begun exploring a very promising alternative by implementing an electrically driven LEDlike system that contains a single quantum dot acting as emitter [70, 103]. They were successful in coupling the quantum dot to a VCSEL-like cavity [104-106], and have recently observed a pronounced Hong-Ou-Mandel dip in the interference of two successively emitted photons $[107,108]$. This new development could open up the route towards versatile on-chip integrated single-photon emitters.

\section{FUTURE DIRECTIONS}

We have been reviewing many different ways of producing single photons from simple quantum systems coupled to optical cavities. Most of the photon-production methods lead to on-demand emission of narrowband and indistinguishable photons into a well defined mode of the radiation field, with efficiencies that can be very close to unity. Therefore these photons are ideal for all-optical quantum computation schemes, as proposed by Knill, Laflamme, and Milburn [2]. Moreover, the atom- and ion-based sources are expected to play a significant role in the implementation of quantum networking [11] and quantum communication schemes [109], as they have already shown to be capable of entangling and mapping quantum states between atoms and photons [9, 10]. Processes like entanglement swapping between distant atoms or ions, that have so far been studied without the aid of cavities $[3,4,110-112]$ are expected to profit enormously from the introduction of cavity-based techniques, as their success-probability scales with the square of the efficiency of the photon generation process. The high efficiency of cavity-based photon sources also opens new routes towards a highly scalable quantum network, which is essential for providing cluster states in one-way quantum computing [113] and for the quantum simulation of complex solid-state systems [114].

\section{Acknowledgments}

We gratefully acknowledge support by the Engineering and Physical Sciences Research Council (EP/E023568/1), the Research Unit 635 of the German Research Foundation, and the EU through the research and training network EMALI (MRTN-CT-2006035369) and the integrated project SCALA. In addition, DL wishes to express his gratitude to the Swedish Research Council (VR) for financial support.
[1] D.P. DiVincenzo, Real and realistic quantum computers, Nature 393 (1998), pp. 113-114.

[2] E. Knill, R. Laflamme, and G.J. Milburn, A scheme for efficient quantum computing with linear optics, Nature 409 (2001), pp. 46-52.

[3] B.B. Blinov et al., Observation of entanglement between a single trapped atom and a single photon, Nature 428 (2004), pp. 153-157.

[4] J. Volz et al., Observation of Entanglement of a Single Photon with a Trapped Atom, Phys. Rev. Lett. 96 (2006), 030404.

[5] S. Olmschenk et al., Quantum Teleportation Between Distant Matter Qubits, Science 323 (2009), pp. 486-489.

[6] E.M. Purcell, Spontaneous Emission Probabilities at Radio Frequencies, Phys. Rev. 69 (1946), p. 681.

[7] H.J. Carmichael, Photon Antibunching and Squeezing for a Single Atom in a Resonant Cavity, Phys. Rev. Lett. 55 (1985), pp. 2790-2793.

[8] F. De Martini et al., Anomalous Spontaneous Emission Time in a Microscopic Optical Cavity, Phys. Rev. Lett. 59 (1987), pp. 2955-2958.

[9] T. Wilk et al., Single-Atom Single-Photon Quantum In- terface, Science 317 (2007), p. 488.

[10] B. Weber et al., Photon-Photon Entanglement with a Single Trapped Atom, Phys. Rev. Lett. (2009), 030501.

[11] J.I. Cirac et al., Quantum State Transfer and Entanglement Distribution Among Distant Nodes in a Quantum Network, Phys. Rev. Lett. 78 (1997), pp. 3221-3224.

[12] D.P. DiVincenzo, The Physical Implementation of Quantum Computation, Fortschr. Phys. 48 (2000), p. 771 .

[13] A.D. Boozer et al., Reversible State Transfer between Light and a Single Trapped Atom, Phys. Rev. Lett. 98 (2007), 193601.

[14] N. Gisin and R. Thew, Quantum Communication, Nature Photonics 1 (2007), pp. 165-171.

[15] E.T. Jaynes and F.W. Cummings, Comparison of Quantum and Semiclassical Radiation Theories with Application to the Beam Maser, Proc. IEEE 51 (1963), pp. 89-109.

[16] B.W. Shore and P.L. Knight, The Jaynes-Cummings Model, J. Mod. Opt. 40 (1993), p. 1195.

[17] N.V. Vitanov et al., Coherent manipulation of atoms and molecules by sequential laser pulses, Adv. At. Mol. 
Opt. Phys. 46 (2001), pp. 55-190.

[18] D.J. Heinzen et al., Enhanced and inhibited spontaneous emission by atoms in a confocal resonator, Phys. Rev. Lett. 58 (1987), pp. 1320-1323.

[19] S.E. Morin, C.C. Yu, and T.W. Mossberg, Strong atomcavity coupling over large volumes and the observation of subnatural intracavity atomic linewidths, Phys. Rev. Lett. 73 (1994), pp. 1489-1492.

[20] L.A. Lugiato, Theory of optical bistability, in Progress in Optics, E. Wolf ed., Vol. XXI Elsevier Science Publishers, B. V., 1984, pp. 71-216.

[21] C.K. Law and J.H. Eberly, Arbitrary Control of a Quantum Electromagnetic Field, Phys. Rev. Lett. 76 (1996), p. 1055.

[22] C.K. Law and H.J. Kimble, Deterministic Generation of a Bit-Stream of Single-Photon Pulses, J. Mod. Opt. 44 (1997), pp. 2067-2074.

[23] A. Kuhn and G. Rempe, Optical Cavity QED: Fundamentals and Application as a Single-Photon Light Source, in Experimental Quantum Computation and Information, F. De Martini and C. Monroe, eds., Vol. 148, IOS-Press, Amsterdam, 2002, pp. 37-66.

[24] A. Kuhn, M. Hennrich, and G. Rempe, StronglyCoupled Atom-Cavity Systems, in Quantum Information Processing, in T. Beth and G. Leuchs, eds., Wiley-VCH, Berlin, 2003, pp. 182-195.

[25] S.E. Harris, Electromagnetically Induced Transparency with Matched Pulses, Phys. Rev. Lett. 70 (1993), pp. $552-555$.

[26] S.E. Harris, Electromagnetically Induced Transparency, Phys. Today 50 (1997), p. 36.

[27] L.V. Hau et al., Light speed reduction to 17 metres per second in an ultracold atomic gas, Nature 397 (1999), pp. $594-598$

[28] D.F. Phillips et al., Storage of Light in Atomic Vapor, Phys. Rev. Lett. 86 (2001), pp. 783-786.

[29] A. Messiah, 1958, Vol. 2, chapter 17. in Quantum Mechanics J. Wiley \& Sons, NY.

[30] A. Kuhn et al., Controlled Generation of Single Photons from a Strongly Coupled Atom-Cavity System, Appl. Phys. B 69 (1999), pp. 373-377.

[31] A.S. Parkins et al., Synthesis of Arbitrary Quantum States Via Adiabatic Transfer of Zeeman Coherence, Phys. Rev. Lett. 71 (1993), p. 3095.

[32] P.P. Rohde, T.C. Ralph, and M.A. Nielsen, Optimal photons for quantum-information processing, Phys. Rev. A 72 (2005), 052332 .

[33] A. Kuhn, M. Hennrich, and G. Rempe, Deterministic single-photon source for distributed quantum networking, Phys. Rev. Lett. 89 (2002), 067901.

[34] M. Keller et al., Continuous generation of single photons with controlled waveform in an ion-trap cavity system, Nature 431 (2004), pp. 1075-1078.

[35] G.S. Vasilev, D. Ljunggren, and A. Kuhn, Single Photons Made-to-Measure, submitted (2009) arXiv:0907.0761v1 [quant-ph].

[36] R.H. Brown and R.Q. Twiss, A test of a new type of stellar interferometer on Sirius, Nature 178 (1956), pp. 1046-1448.

[37] L. Mandel and E. Wolf Optical coherence and quantum optics, Cambridge University Press, Cambridge, UK, 1995.

[38] H.J. Kimble, M. Dagenais, and L. Mandel, Photon Antibunching in Resonance Fluorescence, Phys. Rev. Lett.
39 (1977), pp. 691-695.

[39] H.J. Carmichael et al., Intensity correlations in resonance fluorescence with atomic number fluctuations, J. Phys. A 11 (1978), pp. L121-L126.

[40] F. Diedrich and H. Walther, Nonclassical radiation of a single stored ion, Phys. Rev. Lett. 58 (1987), pp. 203206.

[41] V. Gomer et al., Single-atom dynamics revealed by photon correlations, Phys. Rev. A 58 (1998), pp. R1657R1660.

[42] J. McKeever et al., Experimental realization of a oneatom laser in the regime of strong coupling, Nature 425 (2003), pp. 268-270.

[43] F. De Martini, G.D. Giuseppe, and M. Marrocco, Single-Mode Generation of Quantum Photon States by Excited Single Molecules in a Microcavity Trap, Phys. Rev. Lett. 76 (1996), pp. 900-903.

[44] P. Michler et al., Quantum Correlation Among Photons from a Single Quantum Dot at Room Temperature, Nature 406 (2000), pp. 968-970.

[45] C. Kurtsiefer et al., Stable Solid-State Source of Single Photons, Phys. Rev. Lett. 85 (2000), pp. 290-293.

[46] A. Beveratos et al., Nonclassical radiation from diamond nanocrystals, Phys. Rev. A 64 (2001), 061802.

[47] C. Jurczak et al., Atomic Transport in an Optical Lattice: An Investigation through Polarization-Selective Intensity Correlations, Phys. Rev. Lett. 77 (1996), pp. 1727-1730.

[48] S. Bali et al., Measurements of intensity correlations of scattered light from laser-cooled atoms, Phys. Rev. A 53 (1996), pp. 3469-3472.

[49] M. Hennrich, A. Kuhn, and G. Rempe, Transition from antibunching to bunching in cavity QED, Phys. Rev. Lett. 94 (2005), 053604.

[50] M. Hennrich et al., Photon statistics of a non-stationary periodically driven single-photon source, New J. Phys. 6 (2004), p. 86.

[51] M. Hijlkema et al., A single-photon server with just one atom, Nature Physics 3 (2007), pp. 253-255.

[52] J. McKeever et al., Deterministic Generation of Single Photons from One Atom Trapped in a Cavity, Science 303 (2004), pp. 1992-1994.

[53] C. Santori et al., Indistinguishable photons from a single-photon device, Nature 419 (2002), pp. 594-597.

[54] F. Jelezko et al., Coherence length of photons from a single quantum system, Phys. Rev. A 67 (2003), 041802.

[55] C.K. Hong, Z.Y. Ou, and L. Mandel, Measurement of Subpicosecond Time Intervals between Two Photons by Interference, Phys. Rev. Lett. 59 (1987), pp. 2044-2046.

[56] T. Legero et al., Time-Resolved Two-Photon Quantum Interference, Appl. Phys. B 77 (2003), pp. 797-802.

[57] T. Legero et al., Quantum beat of two single photons, Phys. Rev. Lett. 93 (2004), 070503.

[58] T. Legero et al., Characterization of single photons using two-photon interference, Adv. At. Mol. Opt. Phys. 53 (2006), p. 253.

[59] C.O. Alley and Y.H. Shih, A new type of EPR experiment, in Proceedings of the Second International Symposium on Foundations of Quantum Mechanics in the Light of New Technology, M.Namiki et al. ed., Tokyo, 1986, pp. $47-52$.

[60] T. Wilk et al., Polarization-Controlled Single Photons, Phys. Rev. Lett. 98 (2007), 063601.

[61] P. Michler et al., A Quantum Dot Single Photon Turn- 
stile Device, Science 290 (2000), pp. 2282-2285.

[62] A. Badolato et al., Deterministic Coupling of Single Quantum Dots to Single Nanocavity Modes, Science 308 (2005), pp. 1158-1161.

[63] K. Hennessy et al., Quantum nature of a strongly coupled single quantum dot-cavity system, Nature 445 (2007), pp. 896-899

[64] H.G. Barros et al., Deterministic single-photon source from a single ion, New J. Phys. 11 (2009), 103004.

[65] J. Kim et al., A Single Photon Turnstile Device, Nature 397 (1999), pp. 500-503.

[66] C. Brunel et al., Triggered Source of Single Photons Based on Controlled Single Molecule Fluorescence, Phys. Rev. Lett. 83 (1999), pp. 2722-2725.

[67] B. Lounis and W.E. Moerner, Single Photons on Demand from a Single Molecule at Room Temperature, Nature 407 (2000), pp. 491-493.

[68] R. Brouri et al., Photon Antibunching in the Fluorescence of Individual Color Centers in Diamond, Opt. Lett. 25 (2000), pp. 1294-1296.

[69] C. Santori et al., Triggered Single Photons from a Quantum Dot, Phys. Rev. Lett. 86 (2001), pp. 1502-1505.

[70] Z. Yuan et al., Electrically Driven Single-Photon Source, Science 295 (2002), pp. 102-105.

[71] L.M. Duan, A. Kuzmich, and H.J. Kimble, Cavity QED and quantum information processing with "hot" trapped atoms, Phys. Rev. A 67 (2003), 032305.

[72] Y. Colombe et al., Strong atom-field coupling for BoseEinstein condensates in an optical cavity on a chip, Nature 450 (2007), pp. 272-276.

[73] M. Trupke et al., Atom detection and photon production in a scalable, open, optical microcavity, Phys. Rev. Lett. 99 (2007), 063601.

[74] B. Dayan et al., A Photon Turnstile Dynamically Regulated by One Atom, Science 319 (2008), pp. 1062-1065.

[75] T. Aoki et al., Efficient routing of single photons by one atom and a microtoroidal cavity, Phys. Rev. Lett. 102 (2009), 083601.

[76] M. Pöllinger et al., Ultrahigh-Q tunable whisperinggallery-mode microresonator, Phys. Rev. Lett. 103 (2009), 053901.

[77] A. Rauschenbeutel et al., Step by step engineered many particle entanglement, Science 288 (2000), p. 2024.

[78] A. Wallraff et al., Strong coupling of a single photon to a superconducting qubit using circuit quantum electrodynamics, Nature 431 (2004), p. 162.

[79] X. Maître et al., Quantum Memory with a Single Photon in a Cavity, Phys. Rev. Lett. 79 (1997), pp. 769-772.

[80] S. Brattke, B.T.H. Varcoe, and H. Walther, Generation of Photon Number States on Demand via Cavity Quantum Electrodynamics, Phys. Rev. Lett. 86 (2001), pp. 3534-3537.

[81] F. Brennecke et al., Cavity QED with a Bose-Einstein condensate, Nature 450 (2007), pp. 268-271.

[82] V. Vuletić and S. Chu, Laser cooling of atoms, ions, or molecules by coherent scattering, Phys. Rev. Lett. 84 (2000), pp. 3787-3790.

[83] V. Vuletić, H.W. Chan, and A.T. Black, Threedimensional cavity Doppler cooling and cavity sideband cooling by coherent scattering, Phys. Rev. A 64 (2001), 033405 .

[84] J. McKeever et al., State-insensitive cooling and trapping of single atoms in an optical cavity, Phys. Rev. Lett. 90 (2003), 133602.
[85] P. Maunz et al., Cavity cooling of a single atom, Nature 428 (2004), pp. 50-52.

[86] J.K. Thompson et al., A High-Brightness Source of Narrowband, Identical-Photon Pairs, Science 313 (2006), pp. $74-77$.

[87] K.M. Fortier et al., Deterministic Loading of Individual Atoms to a High-Finesse Optical Cavity, Phys. Rev. Lett. 98 (2007), 233601.

[88] G.R. Guthörlein et al., A single ion as a nanoscopic probe of an optical field, Nature 414 (2001), pp. 49-51.

[89] M. Oxborrow and A.G. Sinclair, Single-Photon Sources, Contemporary Physics 46 (2005), pp. 173-206.

[90] B. Lounis and M. Orrit, Single-Photon Sources, Rep. Prog. Phys. 68 (2005), pp. 1129-1179.

[91] A.J. Shields, Semiconductor quantum light sources, Nature Photonics 1 (2007), pp. 215-223.

[92] O. Benson et al., Regulated and entangled photons from a single quantum dot, Phys. Rev. Lett. 84 (2000), pp. 2513-2516.

[93] E. Moreau et al., Single-mode solid-state single photon source based on isolated quantum dots in pillar microcavities, Appl. Phys. Lett. 79 (2001), pp. 2865-2867.

[94] M. Pelton et al., An Efficient Source of Single Photons: A Single Quantum Dot in a Micropost Microcavity, Phys. Rev. Lett. 89 (2002), 233602.

[95] A.J. Bennett et al., High performance single photon sources from photolithographically defined pillar microcavities, Optics Express 13 (2005), pp. 50-55.

[96] D.C. Unitt et al., Quantum dots as single-photon sources for quantum information processing, Journal of Optics B: Quantum and Semiclassical Optics 7 (2005), pp. S129-S134.

[97] D. Press et al., Photon Antibunching from a Single Quantum-Dot-Microcavity System in the Strong Coupling Regime, Phys. Rev. Lett. 98 (2007), 117402.

[98] M. Benyoucef et al., Enhanced correlated photon pair emission from a pillar microcavity, New J. Phys. 6 (2004), p. 91.

[99] N.H. Lindner and T. Rudolph, Proposal for Pulsed On-Demand Sources of Photonic Cluster State Strings, Physical Review Letters 103(11) (2009), 113602.

[100] A. Kress et al., Manipulation of the spontaneous emission dynamics of quantum dots in two-dimensional photonic crystals, Phys. Rev. B 71 (2005), 241304.

[101] S. Laurent et al., Indistinguishable single photons from a single-quantum dot in a two-dimensional photonic crystal cavity, Appl. Phys. Lett. 87 (2005), 163107.

[102] W. Barnes et al., Solid-state single photon sources: light collection strategies, The European Physical Journal D - Atomic, Molecular, Optical and Plasma Physics 18 (2002), pp. 197-210.

[103] M.B. Ward et al., Electrically driven telecommunication wavelength single-photon source, Appl. Phys. Lett. 90 (2007), 063512.

[104] A.J. Bennett et al., Microcavity single-photon-emitting diode, Appl. Phys. Lett. 86 (2005), 181102.

[105] D.J.P. Ellis et al., Oxide-apertured microcavity singlephoton emitting diode, Appl. Phys. Lett. 90 (2007), 233514.

[106] D.J.P. Ellis et al., Cavity-enhanced radiative emission rate in a single-photon-emitting diode operating at 0.5 GHz, New J. Phys. 10 (2008), 043035.

[107] R.B. Patel et al., Postselective Two-Photon Interference from a Continuous Nonclassical Stream of Photons 
Emitted by a Quantum Dot, Phys. Rev. Lett. 100 (2008), 207405.

[108] A.J. Bennett et al., Indistinguishable photons from a diode, Appl. Phys. Lett. 92 (2008), 193503.

[109] H.J. Briegel et al., Quantum Repeaters: The Role of Imperfect Local Operations in Quantum Communication, Phys. Rev. Lett. 81 (1998), pp. 5932-5935.

[110] B. Sun, M.S. Chapman, and L. You, Atom-photon entanglement generation and distribution, Phys. Rev. A 69 (2004), 042316

[111] J. Beugnon et al., Quantum interference between two single photons emitted by independently trapped atoms, Nature 440 (2006), pp. 779-782.

[112] P. Maunz et al., Quantum interference of photon pairs from two remote trapped atomic ions, Nature Physics 3
(2007), pp. 538-541.

[113] R. Raussendorf and H.J. Briegel, A One-Way Quantum Computer, Phys. Rev. Lett. 86 (2001), pp. 5188-5191.

[114] H.P. Büchler et al., Atomic Quantum Simulator for Lattice Gauge Theories and Ring Exchange Models, Phys. Rev. Lett. 95 (2005), 040402.

[115] We use the term atom throughout the whole paper as a synonym for any quantum system showing discrete energy levels with radiative transitions between them. Amongst these are neutral atoms, ions, quantum dots, SQUIDs, Rydberg atoms and many other artificial quantum systems with atom-like properties.

[116] Note that the population decay rate of the atom is $2 \gamma_{\perp}$, and the photon loss rate from the cavity is $2 \kappa$. 